\title{
Recent Progress in Microfiber-Optic Sensors
}

\author{
Wei LUO ${ }^{1,2}, \mathrm{Ye} \mathrm{CHEN}^{2 *}$, and Fei $\mathrm{XU}^{2 *}$ \\ ${ }^{1}$ School of Electrical and Electronic Engineering, Nanyang Technological University, Singapore 639798, Singapore \\ ${ }^{2}$ National Laboratory of Solid State Microstructures, College of Engineering and Applied Sciences, Nanjing University, \\ Nanjing 210093, China \\ "Corresponding authors: Ye CHEN and Fei XUＥ-mails: yechen@nju.edu.cn and feixu@nju.edu.cn
}

\begin{abstract}
Recently, microfiber-optic sensors with high sensitivity, fast response times, and a compact size have become an area of interest that integrates fiber optics and nanotechnology. Distinct advantages of optical microfiber, such as large accessible evanescent fields and convenient configurability, provide attractive benefits for micro- and nano-scale optical sensing. Here, we review the basic principles of microfiber-optic sensors based on a broad range of microstructures, nanostructures, and functional materials. We also introduce the recent progress and state-of-the-art in this field and discuss the limitations and opportunities for future development.
\end{abstract}

Keywords: Optical microfiber; optical sensing; fiber-optic sensors; microstructures

Citation: Wei LUO, Ye CHEN, and Fei XU, "Recent Progress in Microfiber-Optic Sensors," Photonic Sensors, 2021, 11(1): 45-68.

\section{Introduction}

Since the emergence of the optical fiber endoscope in the first half of the 20th century [1-3], fiber-optic sensors have enabled significant progress in the field of optical sensing [4]. A wide variety of sensor types and applications were demonstrated, which revolutionized optical sensing technology [4]. Current fiber-optic sensors are primarily based on extremely low-loss optical fibers, which were developed from the late 1960s [5, 6]. Optical fibers offer appealing characteristics for sensing applications, including lightweight, large operation bandwidth, immunity to electromagnetic interference, distributing and multiplexing capabilities, biocompatibility, and endurance in harsh environment [4]. Owing to these implementational advantages, fiber-optic sensors, such as fiber-optic gyroscopes [7], fiber-optic hydrophones [8], fiber Fabry-Perot interferometers [9], and fiber gratings [10], have been widely employed in both commercial and military systems $[4,11]$.

Recently, emerging applications in nanotechnology and biology have imposed increasing demands for compact sensors with a smaller footprint, higher sensitivity, faster response, better resolution, and lower power consumption [12, 13]. To meet these imperative requirements, optical microfiber-based sensors have been exploited in the past decades [14-16]. Optical microfiber is a rapidly developing miniaturized waveguide with diameters ranging from tens of nanometers to several micrometers [14, 17]. Compared with standard optical fiber, it provides stronger confinement for guided light while maintaining a low insertion loss $[18,19]$. When the diameter is as low as a subwavelength scale, a significant fraction of light

Received: 27 October 2020 / Revised: 18 November 2020

(C) The Author(s) 2021. This article is published with open access at Springerlink.com

DOI: $10.1007 / \mathrm{s} 13320-021-0614-9$

Article type: Review 
can propagate in the evanescent field outside the physical boundary of the microfiber, which enables strong interactions between the microfiber and its surroundings [19]. These intriguing features make optical microfiber an ideal platform for optical sensing [14, 16, 20-22], near-field coupling [23, 24], atom/particle manipulation $[25,26]$, nonlinear interactions [27-34], and quantum optics [35, 36].

Thus far, a spate of microfiber-optic sensors have been demonstrated for various physical, chemical, and biological parameters (refractive index, temperature, humidity, magnetic field, etc.) $[16,22,37-42]$. This review intends to summarize the recent progress in this field. In Section 2, we review typical microfiber structures that can be utilized for optical sensing. Then, we describe the implementations of microfiber-optic sensors based on different operating mechanisms in Section 3. Finally, in Section 4, we discuss the remaining limitations in the field and conclude with perspectives for the further development of more practical microfiber-optic sensors.

\section{Typical microfiber structures}

\subsection{Optical microfiber}

Optical microfiber typically refers to fiber-optic microwires and nanowires with diameters close to, or below, the wavelength of guided light. The constituent materials of the microfiber can be silica, polymer, and other transparent dielectric media, such as chalcogenide glass [13, 14, 38, 43-48]. This review primarily focuses on silica-based microfiber and its sensing applications. A summary of polymer microfiber sensors can be found in other review articles $[49,50]$.

To fabricate low-loss silica microfibers with excellent uniformity and smoothness, a variety of fabrication methods have been proposed [18, 23, 44, 51-56]. Among the reported methods, the heat-and-pull technique is most frequently adopted because it can fabricate the longest and most uniform microfibers. The technique has been previously used for the manufacture of fiber tapers and fiber couplers $[14,57,58]$. It typically employs a hydrogen flame, which heats and brushes the standard optical fiber that is being stretched. Owing to the pulling force applied by translation stages, the heated part of the fiber elongates, and the diameter gradually reduces. This forms a structure consisting of a stretched waist (the so-called microfiber), two unstretched standard fiber pigtails, and biconical transition sections that link the waist to the pigtails, as shown in Fig. 1. This structure provides ease of measurement because pigtailed ends enable ultralow-loss splicing to standard fiber components (insertion losses $<0.1 \mathrm{~dB}$ ), and the biconical transitions enable highly efficient coupling in and out of the microfiber waist [14]. The length and diameter of the waist and the shape of the transition regions can be precisely defined by controlling the flame movement and stretching parameters. Alternatively, the heating source in a heat-and-pull rig can be replaced by a microheater $[44,56]$ or a sapphire capillary tube heated by a $\mathrm{CO}_{2}$ laser beam [52] to avoid random flame turbulence; this offers a safer fabrication solution without explosive gases. The typical optical loss of a silica microfiber manufactured by the heat-and-pull technique is as low as $\sim 0.01 \mathrm{~dB} / \mathrm{mm}$ [51], owing to the low roughness and high homogeneity.

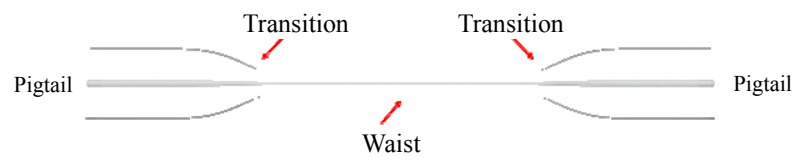

Fig. 1 Typical microfiber structure fabricated from standard optical fiber using the heat-and-pull technique.

Figure 2 shows the ratio of the power propagating in the air to that propagating in the fiber as a function of the microfiber diameter. The ratio increases with a decreasing diameter, indicating a larger fraction of power in the evanescent field for thinner microfibers. When the diameter reaches $0.5 \mu \mathrm{m}$, nearly $94 \%$ of the total light energy is outside the microfiber, i.e., in the surrounding environment. Additional to the properties of large 
evanescent fields, strong optical confinement, and tailorable waveguide dispersion, optical microfiber also provides excellent mechanical strength and flexibility [59]. Bending radii of a few micrometers have been achieved with low induced bending losses $[60,61]$. The robustness and configurability of optical microfibers enable various compact microfiber structures, e.g., loops [52, 62, 63], knots $[18,64,65]$, coils [66-70], couplers [71-76], and interferometers [77-86]. Moreover, gratings [21, 87-89] and functional materials [13, 38] can also be integrated with microfibers to enhance the functionalities of the platform. These structures will be discussed in the following sections.

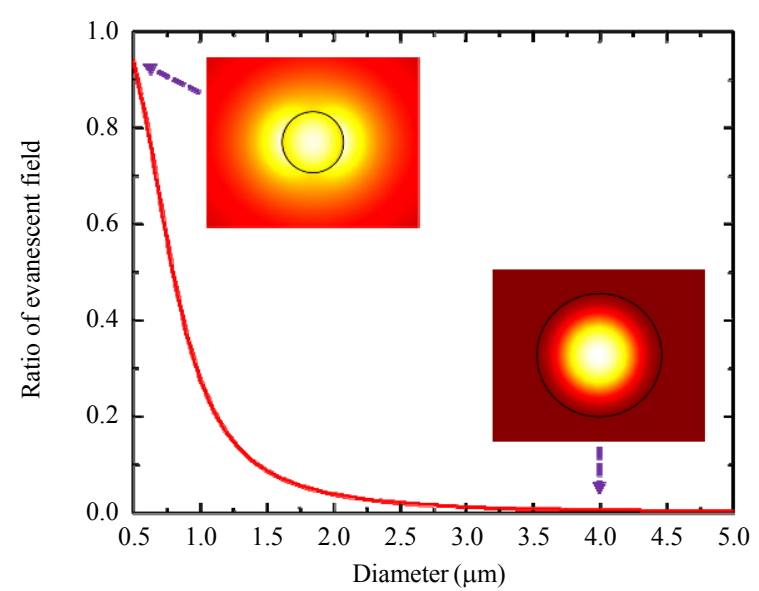

Fig. 2 Ratio of evanescent field penetrating into surrounding air to the field propagating in the fiber as a function of the fiber diameter at a wavelength of $1550 \mathrm{~nm}$. The upper and lower insets are the power flow distributions of $0.5-\mu \mathrm{m}$-diameter and $4-\mu \mathrm{m}$-diameter silica microfibers, respectively. Dimensions of the insets are not to scale.

\subsection{Microfiber coupler}

A fiber coupler is a basic fiber optics device, which has been intensively studied [73, 90, 91]. Recently, it has attracted renewed attention, owing to the development of optical microfiber [74]. A microfiber coupler (MFC) is based on evanescent coupling between two adjacent microfibers. Its typical structure is shown in Fig. 3. For an MFC with a large index-contrast and two waveguides in physical contact, conventional perturbation theory cannot be applied. Thus, the supermode theory is employed to describe the MFC [92]. As shown in
Fig. 3, if light is launched into Port 1, the output powers from Ports 3 and 4 are given by

(a)

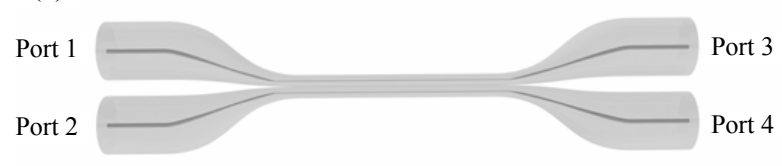

(b)

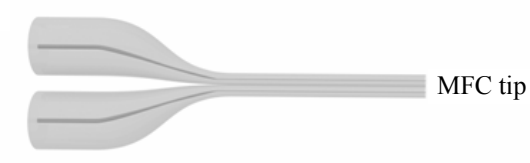

(c)

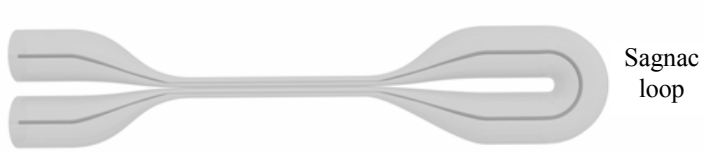

Fig. 3 Schematic of optical microfiber coupler (MFC) structures: (a) typical structure of MFC, (b) MFC probe, manufactured by cleaving the waist, and (c) Sagnac loop mirror, manufactured by fusing the MFC.

$$
\begin{aligned}
& P_{3}^{i}=P_{1}^{i} \cos ^{2}\left(\frac{\pi L \Delta n_{\mathrm{eff}}^{i}}{\lambda}\right) \\
& P_{4}^{i}=P_{1}^{i} \sin ^{2}\left(\frac{\pi L \Delta n_{\mathrm{eff}}^{i}}{\lambda}\right) \\
& \Delta n_{\mathrm{eff}}^{i}=n_{\mathrm{eff}}^{i, \text { even }}-n_{\mathrm{eff}}^{i, \text { dd }}
\end{aligned}
$$

where $i$ indicates transverse electric (transverse magnetic) polarization, $P_{1}^{i}$ is the input power, $L$ is the coupling length, $\Delta n_{\text {eff }}^{i}$ is the effective refractive index (RI) difference between $n_{\text {eff }}^{i, \text { even }}$ for the even supermode and $n_{\text {eff }}^{i \text {,odd }}$ for the odd supermode, and $\lambda$ is the optical wavelength. From the formulas, one can observe that the MFC outputs are strongly dependent on the variation of $\Delta n_{\text {eff }}^{i}$, which is caused by changes in the physical parameters of its surroundings, such as temperature and strain. Therefore, it is an attractive structure for highly sensitive optical sensing. The sensing parameters can be estimated by either monitoring the spectrum shift [71, 93] or the transmittance at selected wavelengths [94]. In addition, the MFC can operate as a microprobe $[72,75,95]$, if its waist is cleaved, or a Sagnac loop [96-98], if output ports 3 and 4 are fused, as shown in Figs. 3(b) and 3(c), respectively.

The RI sensitivity $(S)$ of the MFC can be defined 
as $[99,100]$

$$
S=\frac{\partial \lambda_{s}}{\partial n_{a}}=\frac{\lambda_{s}}{\Delta n_{\mathrm{eff}}^{i}-\lambda_{s} \partial\left(\Delta n_{\mathrm{eff}}^{i}\right) / \partial \lambda} \frac{\partial\left(\Delta n_{\mathrm{eff}}^{i}\right)}{\partial n_{a}}
$$

where $\lambda_{s}$ is the wavelength of the dip in the MFC transmission spectrum, $n_{a}$ is the $\mathrm{RI}$ of the surrounding medium, and $g^{i}=\Delta n_{\text {eff }}^{i}-\lambda_{s} \partial\left(\Delta n_{\text {eff }}^{i}\right) / \partial \lambda$ is the group index difference between the even and odd supermodes. According to this equation, the RI sensitivity approaches infinity when $g^{i}=0$ at the turning point, which is an important characteristic that has been applied to enhance RI sensing [99, 100].

\subsection{Microfiber interferometer}

Interferometers are among the most broadly used structures for high-sensitivity optical sensing [101-103]. Microfiber interferometers (MFIs) have been demonstrated recently; these interferometers are based on evanescent coupling [77], birefringent interference $[79,80]$, and multimode interference [81-85]. Figure 4 shows the basic structures of a microfiber Mach-Zehnder interferometer (MZI) and modal interferometers. The operating principle of MFIs can be explained by dual-beam interference, and the transmission intensity is given by [38]

$$
\begin{gathered}
I=I_{1}+I_{2}+2 I_{1} I_{2} \cos \Delta \varphi \\
\Delta \varphi=\frac{2 \pi}{\lambda}\left(n_{1, \mathrm{eff}} L_{1}-n_{2, \mathrm{eff}} L_{2}\right)
\end{gathered}
$$

where $I_{1}$ and $I_{2}$ are the intensities of the two light beams in respective arms, polarizations, or modes, and $\Delta \varphi$ is the phase difference between them. The interference fringe in a transmission spectrum and the sensing parameters can be demodulated by a shift in the fringe. Compared with conventional waveguide MZIs, microfiber MZIs can provide a sensitivity that is one order of magnitude higher and a smaller footprint between tens and hundreds of micrometers [20]. The microfiber modal interferometers fabricated from single-mode fiber [81, 84-86, 99, 104, 105], microstructured fiber [83,
106-108], and multimode fiber [82, 109, 110] also provide the advantages of compactness, high sensitivity, and simple fabrication.

(a)

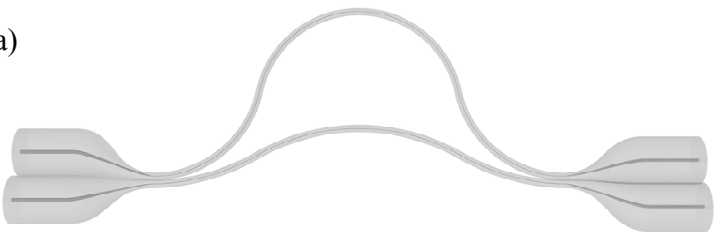

(b)

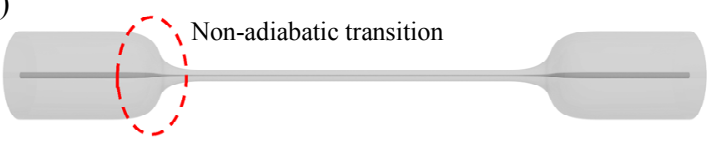

(c)

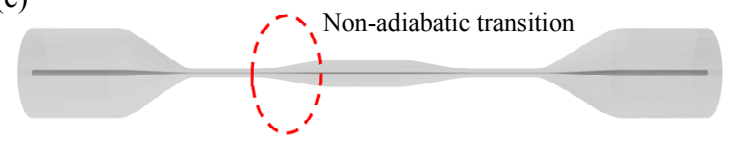

Fig. 4 Schematic of MFI structures: (a) microfiber Mach-Zehnder interferometer (MZI); (b-c) microfiber modal interferometers with non-adiabatic transitions.

\subsection{Microfiber grating}

A fiber grating is a periodic structure that modulates the effective RI along the optical fiber [111]. Since the first successful fabrication in 1978 [112], it has been widely utilized for optical filtering and sensing [10, 113]. A typical fiber Bragg grating (FBG) is several millimeters in length and has a diameter of approximately $100 \mu \mathrm{m}$. Its relatively large size limits its applications in RI sensing and the detection of ultrasmall objects [21]. Owing to the large evanescent field induced by a small diameter and high RI contrast, microfiber gratings have become a powerful tool to overcome this limitation and have received increasing interest in recent years [21, 114]. Many microfiber gratings have been demonstrated using various techniques, including chemical etching $[115,116]$, laser irradiation/ ablation [117-121], focused ion beam milling [122-128], lithography [129, 130], and external modulations [87, 131-133]. Compared with the conventional fiber, the high modulation of the effective RI $\left(10^{-3}-10^{-1}\right)$ in microfibers enables ultra-compact gratings with lengths between 
$10 \mu \mathrm{m}$ and $100 \mu \mathrm{m}$ [21]. There are primarily two types of gratings in microfiber: the microfiber Bragg grating (MFBG) and the microfiber long period grating (MFLPG). For the MFBG, when the forward-propagating mode is coupled with the identical backward mode, the first-order diffraction can be described by the Bragg resonance condition [111]:

$$
\lambda_{B}=2 n_{\text {eff }} \Lambda
$$

where $\lambda_{B}$ is the Bragg wavelength, $n_{\text {eff }}$ is the effective RI of the guided mode, and $\Lambda$ is the period of the MFBG. Any changes in the surrounding parameters that affect $n_{\text {eff }}$ and $\Lambda$ will finally result in a shift in the Bragg wavelength. For the MFLPG with a forward-propagating mode coupled into higher order modes in the same direction, a similar resonance condition can be obtained by considering the phase matching between coupled modes [111]:

$$
\lambda_{L}=\left(n_{\mathrm{eff}, 1}-n_{\mathrm{eff}, 2}\right) \Lambda=\Delta n_{\mathrm{eff}} \Lambda
$$

where $\Delta n_{\text {eff }}=n_{\text {eff, } 1}-n_{\text {eff }, 2}$ is the modal effective RI difference. According to (8), the MFLPG has a significantly longer period than that of the MFBG; thus, its fabrication requirements are lower.

\subsection{Microfiber resonator}

As illustrated in Fig. 5, a variety of resonance structures can be implemented using optical microfibers, including a loop [52, 62, 63], a knot [18, 64, 65], coil [66-70], a photonic crystal $(\mathrm{PhC})$ cavity $[122,125,134-137]$, a ring $[138,139]$, a Fabry-Perot (FP) cavity [140-142], and their extended configurations [143-147]. A typical microfiber resonator has a quality $(Q)$ factor ranging from $10^{2}$ to $10^{6}$, depending on the specific structures $[13,38]$. The high $Q$ factor enables its application in sensors, lasers, dynamic filters, optical delay lines, and quantum optics [38].

Considering a microfiber loop resonator as an example, the resonance condition is [148]

$$
\lambda_{c}=\frac{n_{\text {eff }} L}{m}
$$

(a)

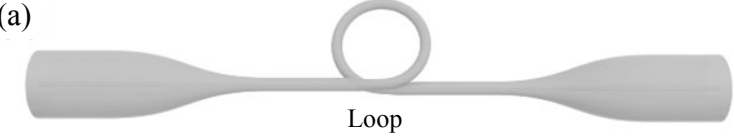

(b)

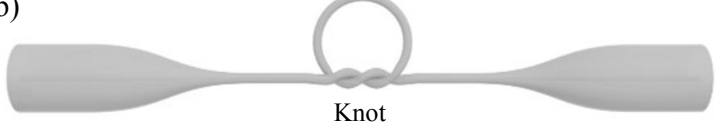

(c)

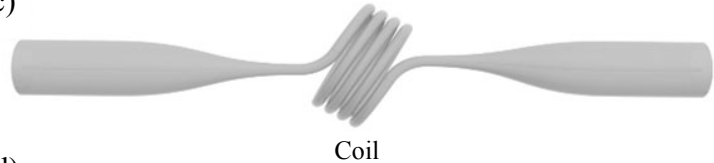

(d)

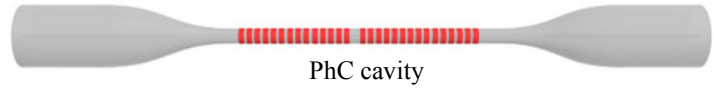

Fig. 5 Schematic of typical microfiber resonators: (a) microfiber loop resonator, (b) microfiber knot resonator, (c) microfiber coil resonator, and (d) microfiber PhC cavity.

where $\lambda_{c}$ is the resonant wavelength, $L$ is the circumference of the loop, and $m$ is an integer representing the resonance order. According to (9), the RI sensitivity, obtained by monitoring the shift of the resonant wavelength, can be expressed as [148]

$$
S=\frac{\partial \lambda_{c}}{\partial n_{a}}=\frac{\partial \lambda_{c}}{\partial n_{\text {eff }}} \frac{\partial n_{\text {eff }}}{\partial n_{a}}=\frac{\lambda_{c}}{n_{\text {eff }}} \frac{\partial n_{\text {eff }}}{\partial n_{a}}
$$

which only depends on the change in the RI of the surrounding medium, regardless of the resonator's $Q$ factor. However, the detection limit, which is an important figure of merit for optical sensors, may benefit from the high $Q$ factor of microfiber resonators [68, 149].

\subsection{Functional material-integrated microfiber devices}

In addition to geometric structures, it is also an effective strategy to integrate functional materials into microfiber platforms to extend their functionalities. Owing to the large evanescent fields with high surface intensity, functional material-integrated microfiber devices offer strong light-matter interactions for sensing applications. Various aforementioned microfiber structures have been reported for integration with plasmonic materials [150-156], polymers [49, 157-160], 
two-dimensional (2D) materials [161-173], sol-gels [174, 175], magnetic fluids [176-178], and biomaterials [41]. For example, Fig. 6 shows the typical configurations of hybrid graphene-fiber structures, including a free-standing microfiber, microfiber on a low-RI substrate, and a microfiber knot resonator on a low-RI substrate. These composite structures of 2D materials and microfibers are realized by the wet transfer method that is summarized in the review article [179]. The extremely high surface-volume ratio and ultrafast response of graphene provide useful characteristics for chemical molecular sensing and all-optical control [180, 181].

(a)

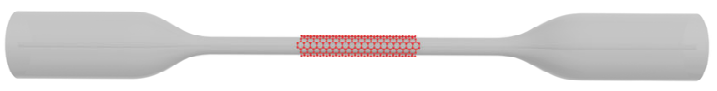

(b)

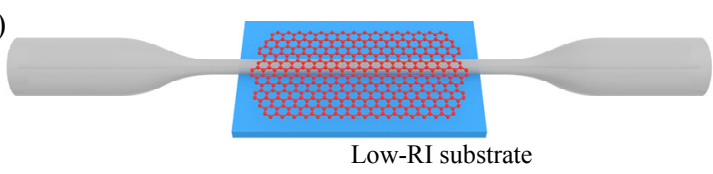

(c)

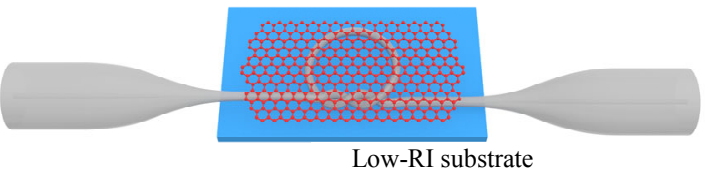

Fig. 6 Graphene functionalized optical fiber platform, presented as an example. Functional materials can be integrated with (a) a free-standing microfiber, (b) a microfiber on a low-RI substrate, and (c) a microfiber knot resonator on a low-RI substrate.

\section{Microfiber-optic Sensors}

Owing to favorable features, such as compact size and high sensitivity, a variety of microfiber-based optical sensors have been developed. Figure 7 summarizes the basic sensing principles of these sensors. According to these principles, most microfiber sensors can be classified into three categories. First, interference and resonance effects of microfiber structures lead to phase-based sensors. This type of sensor is extremely sensitive to phase changes resulting from
RI variation induced by surrounding medium alteration, thermal-optic or elasto-optic effects, and deformation caused by thermal expansion or externally applied forces. Information of miscellaneous measurands can be easily demodulated by monitoring the shift of the interference fringe and resonance dip or recording the intensity at specific wavelengths. Second, optical absorption, leaky radiation, and optical elastic scatterings enable loss-based microfiber sensors. Such sensors employ absorption spectroscopy, optical transmittance measurements, and optical microscopy in the analysis and characterization of gases, liquids, nanoparticles, and chemical or biological molecules. Selective sensing with enhanced performance can be realized by the integration of appropriate functional materials. Third, the newly generated frequency spectral components from optical inelastic scatterings, parametric and non-parametric nonlinear processes, and fluorescence contribute to frequency-based microfiber sensors. The strong optical confinement and large evanescent fields of the sensors enable ultra-sensitive scattering, nonlinear, and fluorescence spectroscopies for the investigation of various physical, chemical, and biological measurands. In the following sections, typical microfiber-optic sensors and their recent progress will be introduced in terms of the working principle classification.

\subsection{Phase-based sensors}

Phase-based microfiber sensors that utilize interference and resonance effects have been widely studied because they exhibit simplicity and convenience in manufacturing and implementation. All the aforementioned microfiber structures, including couplers, MZIs, modal interferometers, gratings, and the various types of resonators, have been employed as sensing elements for a range of applications. In many cases, the variation in 
interference and resonance conditions are caused by the effective RI change induced by the change of the surrounding RI. Thus, an effective sensitivity can be defined to describe the performance of phase-based microfiber sensors [22]:

$$
S_{\text {eff }} \propto \frac{\partial n_{\text {eff }}}{\partial n_{a}} .
$$

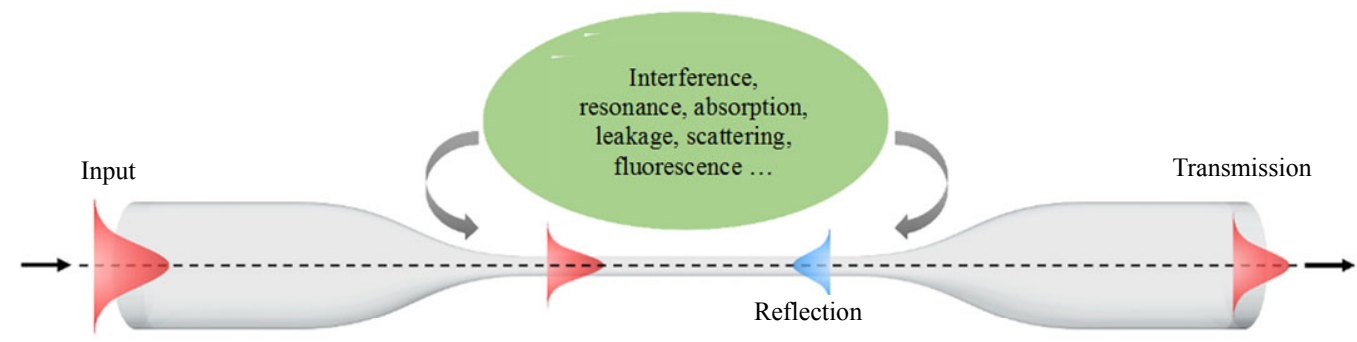

Fig. 7 Basic sensing processes of microfiber-optic sensors. By detecting and analyzing variations in optical phase, intensity, frequency, and polarization of the transmitted and reflected light signals from microfiber structures, the surrounding physical, chemical, and biological parameters can be demodulated regarding the detailed interaction processes. Diverse microfiber structures provide versatile platforms for various physical, chemical, and biological effects with favorable characteristics, such as a small footprint, strong optical confinement, and large evanescent fields. The green ellipse represents the typical physical effects that constitute the operating principles of microfiber-optic sensors.

The effective sensitivity is correlated to the microfiber diameter and the range of the surrounding RI. As illustrated in Fig. 8, a phase-based sensor exhibits a higher effective sensitivity with a decrease in the microfiber diameter or an increase in the surrounding RI. This feature is caused by the larger evanescent fields in smaller dimensions or reduced RI contrast, indicating that microfiber sensors with smaller diameters would benefit sensing applications.

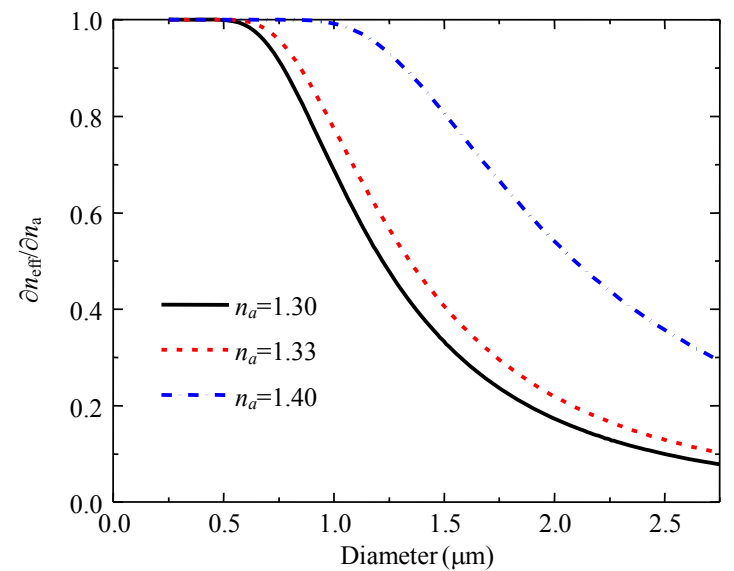

Fig. 8 Effective sensitivity of a phase-based silica microfiber sensor as a function of diameter for different surrounding refractive indices at a wavelength of $1550 \mathrm{~nm}$.
To date, MFCs have been widely demonstrated in the sensing of RIs [96, 100, 182], temperature [72, 75, 183, 184], strain/force [97], current [94], magnetic fields [94, 176], humidity [169, 185, 186], and other parameters. Most of the reported MFC RI sensors exhibited sensitivities in the range of $1000 \mathrm{~nm} / \mathrm{RIU}-6000 \mathrm{~nm} / \mathrm{RIU}$ (RI unit) [93, 95, 96, 187, 188]. Ultrahigh sensitivity is possible when the MFC operates near the turning point [100] or relies on the birefringence-induced Vernier effect [182] (Fig. 9). Using a Sagnac loop mirror based on an MFC, Chen et al. implemented a highly sensitive reflective micro-force sensor with a maximum sensitivity of $\sim 3754 \mathrm{~nm} / \mathrm{N}$ [97]. An MFC sensor was integrated with molybdenum disulfide $\left(\mathrm{MoS}_{2}\right)$ nanosheets and implemented for the simultaneous measurement of relative humidity (RH) and temperature with an RH sensitivity of $115.3 \mathrm{pm} / \% \mathrm{RH}$ in the range of $54.0 \% \mathrm{RH}-93.2 \% \mathrm{RH}$ and a temperature sensitivity of $-104.8 \mathrm{pm} /{ }^{\circ} \mathrm{C}$ in the range of $30^{\circ} \mathrm{C}-90^{\circ} \mathrm{C}$ [169]. 


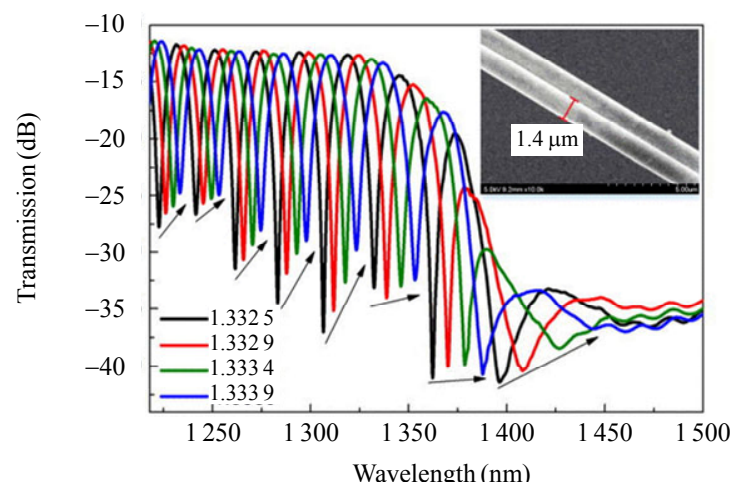

(a)

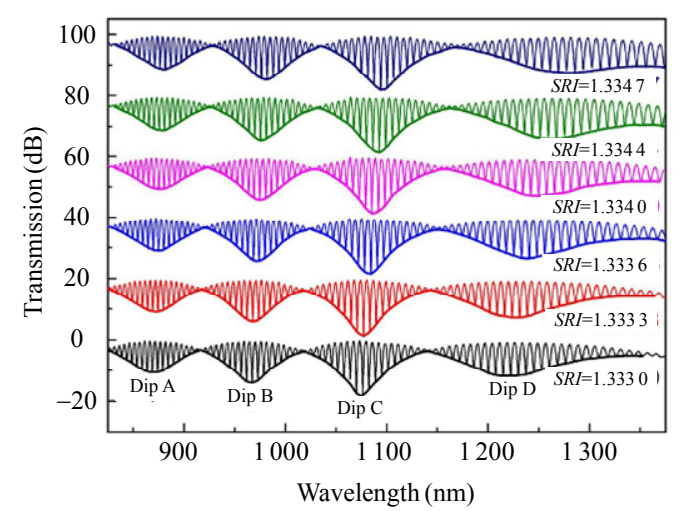

(c)

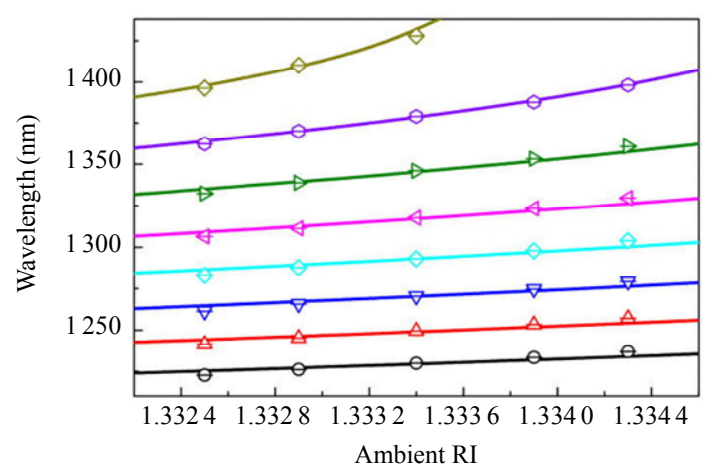

(b)

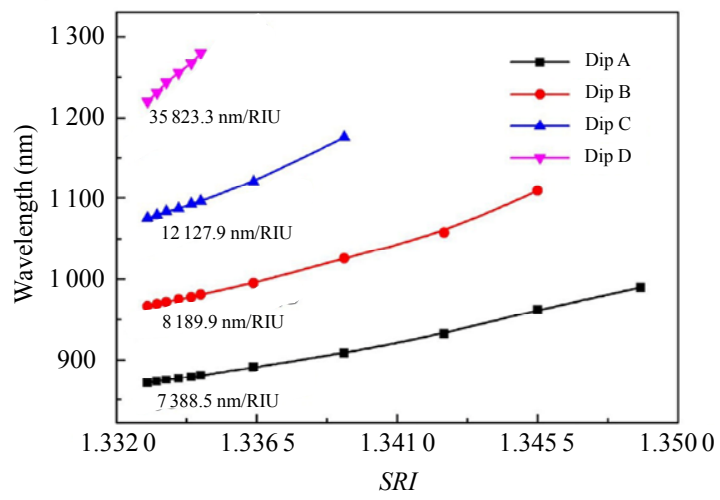

(d)

Fig. 9 Phase-based MFC sensors with ultrahigh sensitivities: (a) transmission spectral responses to different ambient RIs for an MFC sensor operating near the turning point of effective group index difference. Reproduced with permission [100]. Copyright 2016 AIP Publishing; (b) modeled (solid curves) and measured wavelengths (points) at transmission dips versus ambient RI for the MFC sensor operating near the turning point. Reproduced with permission [100]. Copyright 2016 AIP Publishing; (c) transmission spectra of an MFC sensor with the Vernier effect for different surrounding RIs (spectra are offset by $22 \mathrm{~dB}$ ). Reproduced with permission [182]. Copyright 2018 Elsevier; (d) measured wavelengths at transmission dips versus surrounding RI for the MFC sensor with the Vernier effect. Reproduced with permission [182]. Copyright 2018 Elsevier.

MFIs also exhibit excellent performance in phase-sensitive optical sensing. For example, Wo et al. [78] demonstrated an MZI using a 2- $\mu \mathrm{m}$ silica microfiber as the sensing arm, with an RI sensitivity of $7159 \mu \mathrm{m} /$ RIU. Excluding MZIs with different optical paths, modal interferometers comprising multimode microfiber [99] and non-adiabatic transition regions [189, 190] have been demonstrated for RI, gas, and liquid level sensing. Sun et al. [80] reported a highly birefringent microfiber loop interferometer with an RI sensitivity of approximately $24373 \mathrm{~nm} / \mathrm{RIU}$ and a temperature stability above $0.005 \mathrm{~nm} /{ }^{\circ} \mathrm{C}$. Moreover, based on graphene-MFI hybrid structure, Yao et al. [191] realized an all-optical $\mathrm{NH}_{3}$ gas sensor with a high sensitivity of $\sim 6 \mathrm{pm} / \mathrm{ppm}$ and a resolution of $\sim 0.3 \mathrm{ppm}$ as shown in Figs. 10(a) and 10(b). A cascaded MFI structure is another interesting topic, owing to its ability to demodulate multiple sensing parameters [192-194]. A multimode microfiber-based dual MZI can achieve simultaneous measurements of RI and temperature with sensitivities of $2576.584 \mathrm{~nm} / \mathrm{RIU}$ and $1001.864 \mathrm{~nm} / \mathrm{RIU}$ and $-0.193 \mathrm{~nm} /{ }^{\circ} \mathrm{C}$ and $0.239 \mathrm{~nm} /{ }^{\circ} \mathrm{C}$, respectively [192]. Recently, a microfiber modal interferometer was functionalized with glucose oxidase and proposed for bio-selective and high-sensitivity glucose detection with a 
response coefficient of $1.74 \mathrm{~nm} / \mathrm{mg}^{-1} \cdot \mathrm{ml}^{-1}$, as shown in Figs. 10(c) and 10(d) [195].

MFBGs for RI [119, 121, 123, 128, 129], temperature [124, 127, 196], strain [196, 197], force [197, 198], and humidity [199] sensing have been reported; the large evanescent fields enable high RI sensitivities of $10^{2} \mathrm{~nm} / \mathrm{RIU}-10^{3} \mathrm{~nm} / \mathrm{RIU}$. However, the temperature and strain sensitivities of MFBGs have not been improved compared with those of bulk FBGs. MFBGs exhibit an advantage over bulk FBGs for force sensing, particularly for detections of micro-force because their reduced thickness significantly enhances the sensitivity $\left(10^{2} \mathrm{~nm} / \mathrm{N}-\right.$ $10^{3} \mathrm{~nm} / \mathrm{N}$ ). For practical applications, it is beneficial to realize the simultaneous measurement of multiple parameters and avoid cross-sensitivity [196]. For this purpose, Lee et al. [200] developed a multimode etched-core FBG sensor with an asymmetric non-adiabatic taper and demonstrated simultaneous demodulation of the RI, temperature, and strain with accuracies of $1 \times 10^{-4}$ RIU, $0.32{ }^{\circ} \mathrm{C}$, and $10 \mu \varepsilon$, respectively. The functionality of an MFBG sensor can be enhanced by functional material coatings. For example, an MFBG coated with the graphene oxide (GO) film was implemented for relative humidity sensing with a sensitivity of $17.361 \mathrm{pm} / \mathrm{RH} \%$ and linear correlation coefficient of $99.89 \%$ [199]. In addition to MFBGs, MFLPGs were also implemented for optical sensing [87, 88, 201, 202], exhibiting a comparable performance with MFBGs, while requiring a simpler manufacturing process $[87$, 88, 117, 201-203]. (a)

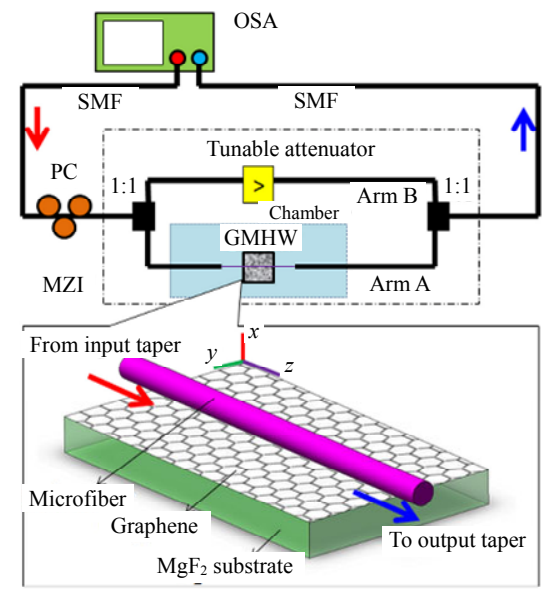

(c)

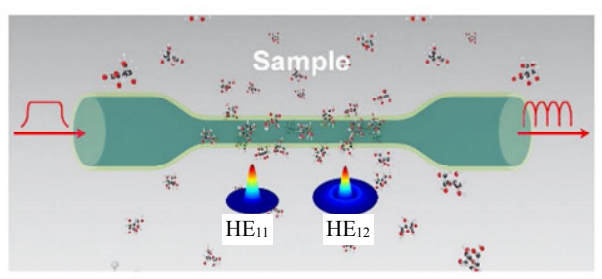

(b)
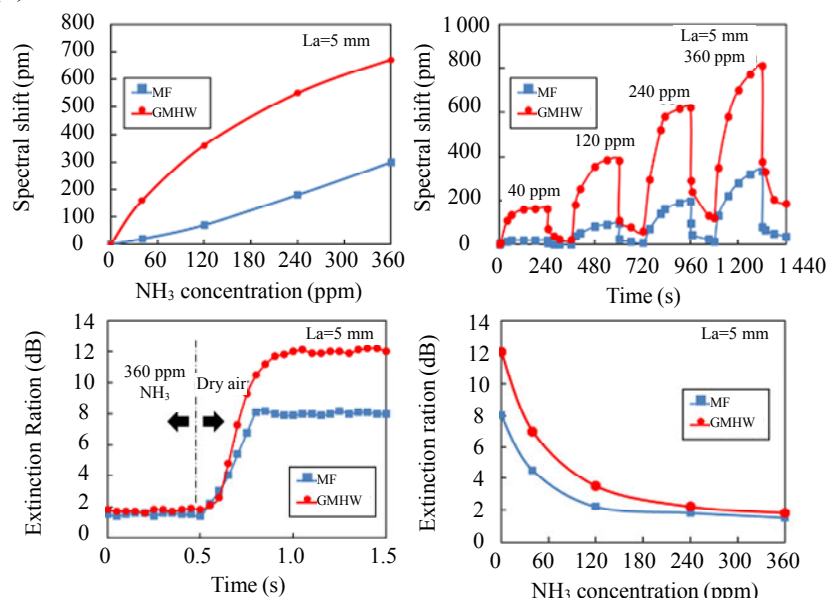

(d)

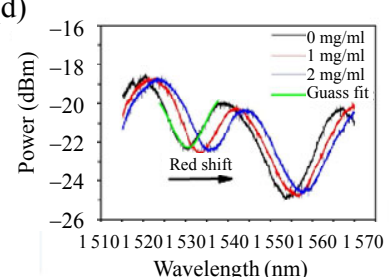

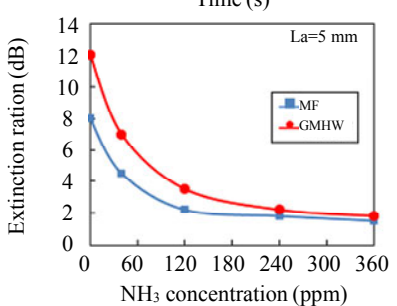

(e)

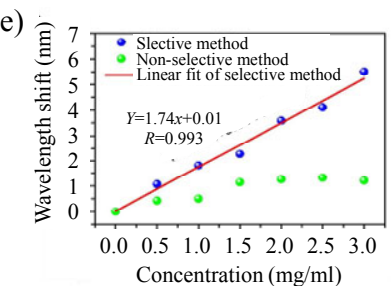

Fig. 10 Phase-based MFI sensors: (a) schematic diagram of a graphene/microfiber hybrid waveguide (GMHW) and setup of the GMHW-MZI for $\mathrm{NH}_{3}$ sensing. Reproduced with permission [191]. Copyright 2014 Elsevier; (b) sensing performances of the GMHW (red) and the microfiber on $\mathrm{MgF}_{2}$ without graphene attached (blue). Reproduced with permission [191]. Copyright 2014 Elsevier; (c) schematic diagram of a functionalized microfiber modal interferometer for selective and highly sensitive glucose detection. Reproduced with permission [195]. Copyright 2018 Elsevier; (d) spectra of the functionalized MFI in glucose solutions with different concentrations. Reproduced with permission [195]. Copyright 2018 Elsevier; (e) relationship between resonant wavelength shift and concentration for the functionalized MFI. Reproduced with permission [195]. Copyright 2018 Elsevier.

Owing to their compact size, high $Q$ factor, and easy coupling strategy, microfiber-based resonant sensors have been widely applied in the measurement of the RI $[68,69,139,140,146,147$, 204], temperature [63, 141, 205-209], current [210, 211], electric field [212], magnetic field [178], and 
humidity [213]. For example, as the simplest microfiber resonant structure, a microfiber loop resonator with a $Q$ factor of $\sim 10^{5}$ has been demonstrated for temperature sensing with $\sim 0.1 \mathrm{mK}$ resolution [63]. However, the freestanding microfiber loop maintained by van der Waals and electrostatic forces is weak in mechanical stability. Alternatively, microfiber resonators based on more stable structures, such as a knot [178, 205, 208, 209, $212,213]$, a coil [68, 69, 211, 214, 215], a ring [139], and an FP cavity [140, 141], have been proposed and implemented for sensing applications. An encapsulation process was also employed to stabilize the freestanding microfiber resonance structures [70, 216]; however, the packaging will influence the Q factor and field overlap between the structure and the analyte [217].

\subsection{Loss-based sensors}

Ultrasensitive microfiber absorption spectroscopy can be utilized for molecule detections [218, 219], owing to the large evanescent fields and strong light-matter interactions in subwavelength microfibers. For microfiber sensors based on absorption effects, the transmission loss depends on the fraction of the evanescent fields, molecular concentration, absorptivity, and effective interaction length [38]. To obtain high sensitivity, microfiber structures, such as optical resonators, can be introduced to enhance the overlap between guided light and the surroundings or increase the effective interaction length [220, 221]. Furthermore, functional material coatings, such as metal nanostructures [222-224], antibodies [224, 225], gelatin [226], doped sol-gel films [227], and graphene [228-230], have been adopted for the realization of selective sensing. Recently, an absorption-based strain sensor using a graphene-microfiber hybrid structure was reported [231]. The optical conductivity of graphene on a microfiber can be tuned using external strain applied on the microfiber, resulting in a change of transmission intensity in the spectrum [232, 233]. Sun et al. [234] showed that the absorption of the graphene-assisted structure was also sensitive to the surrounding temperature. As illustrated in Figs. 11(a) and 11(b), Chen et al. [170] demonstrated that the absorption edge of the monolayer $\mathrm{WS}_{2}$ on a microfiber linearly shifted with the uniaxial strain, exhibiting a sensitivity of $\sim 10 \mathrm{~nm} / \%$ strain.

A microfiber sensor based on radiation modes was developed for conditions where the surrounding RI is larger than that of the microfiber. Gao et al. [236] demonstrated the possibility of employing leaky radiation to measure an environment RI that was higher than that of the microfiber material with a theoretically predicted sensitivity higher than $400 \%$ RIU. Recently, Zhang et al. [237] reported a sensor that enabled multifunctional flow sensing in microfluidic chips, which utilized the transition from guided to radiation modes for a microfiber embedded in polydimethylsiloxane film.

Owing to the strong optical confinement and large evanescent fields, elastic light scatterings are significantly enhanced in a microfiber. For example, Polynkin et al. [238] reported a geometric scattering-based RI sensor by integrating a microfiber with a microfluidic channel; the sensor realized an estimated resolution of $\sim 10^{-4}$ RIU. Liu et al. [239] proposed a Mie scattering-based microfiber RI sensor with an accuracy of $1.8 \times 10^{-5}$ RIU. Rayleigh scattering is one of the most important elastic scattering effects and has been widely studied in microfibers because rapid detection and evaluation of nanoparticles are important in the fields of nanoscience and nanotechnology [235, 240-246]. Wang et al. [242] theoretically investigated nanoparticle-induced Rayleigh-Gans scattering in microfibers for optical sensing. By measuring the additional loss introduced by the scattering of microparticles, Wei et al. [246] implemented a microparticle sensor by simply using a microfiber. Chen et al. [244] demonstrated that the detection limit for a single nanoparticle can be enhanced by utilizing a hybrid plasmonic-photonic mode in a subwavelength microfiber. Yu et al. [245] 
realized single-nanoparticle detection and sizing by a microfiber pair in an aqueous environment. Recently, they have further demonstrated an
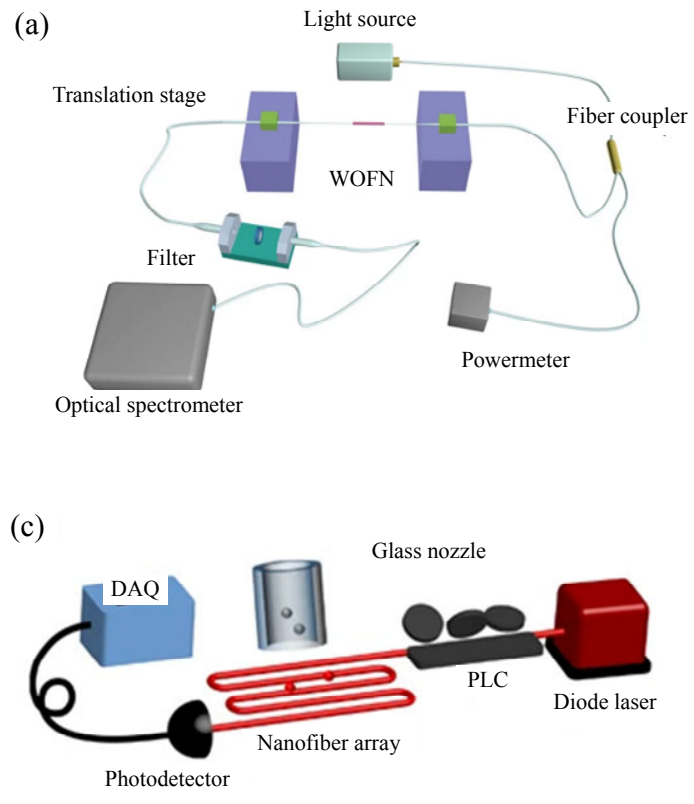

accurate evaluation of the distribution of ultrafine particulate matter in air using a microfiber array as shown in Figs. 11(c) and 11(d) [235].

(b)

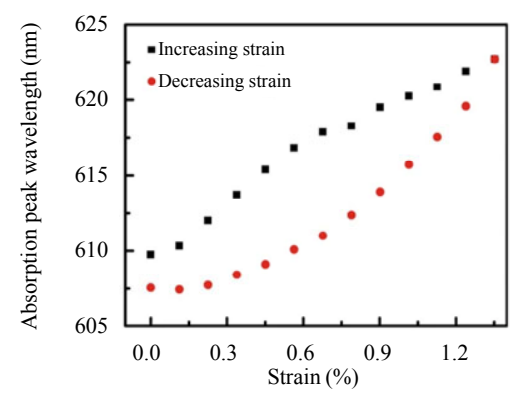

(d)

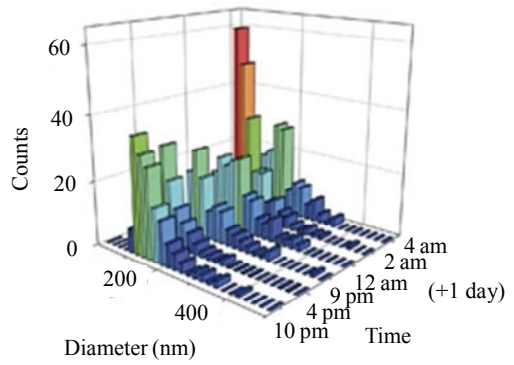

Fig. 11 Loss-based microfiber sensors: (a) experimental setup for in-line strain manipulation of absorption spectra of monolayer $\mathrm{WS}_{2}$ to an optical fiber nanowire (WOFN). Reproduced with permission [170]. Copyright 2019 Springer Nature; (b) absorption peak wavelength of the WOFN with the increase and decrease in the strain. Reproduced with permission [170]. Copyright 2019 Springer Nature; (c) schematic setup of a Rayleigh-Gans-scattering-based nanofiber array size spectrometry. DAQ, data acquisition system; PLC, polarization controller. Reproduced with permission [235]. Copyright 2018 Springer Nature; (d) size histogram of nanoparticles in six air samples collected from different moments, evaluated by the nanofiber array size spectrometer. Reproduced with permission [235]. Copyright 2018 Springer Nature.

\subsection{Frequency-based sensors}

In addition to elastic light scattering, the abundant inelastic scattering effects in microfibers also provide useful tools for sensing applications. Recently, Brillouin scattering in microfiber has attracted increasing interest because the microfiber can strongly confine both optical and acoustic modes at a nanoscale and provide a unique platform to investigate photon-phonon interactions [247-253]. For optical sensing, Brillouin scattering may benefit the sensitivity and compactness of microfiber sensors, owing to the rise of surface acoustic waves. Godet et al. [254] characterized subwavelength microfibers with a resolution of a few nanometers using Brillouin spectroscopy. Huang et al. [255] demonstrated a microfiber Brillouin sensor with a maximum pressure sensitivity of $0.066 \mathrm{MHz} / \mathrm{kPa}$ and a temperature sensitivity two times higher than that of a standard fiber-based Brillouin sensor. Luo et al. [256] revealed strain sensitivities of $0.0086 \mathrm{MHz} / \mu \varepsilon$ and $0.020 \mathrm{MHz} / \mu \varepsilon$ for the axially symmetric $\mathrm{R}_{01}$ and $\mathrm{R}_{02}$ acoustic modes in a microfiber, respectively. Recently, Huang et al. [257] demonstrated an RI sensor utilizing Brillouin scattering in a microfiber with a $2-\mu \mathrm{m}$ diameter with an RI sensitivity of $\sim 1.6 \mathrm{GHz} / \mathrm{RIU}$.

Intrinsic Raman scattering in bulk optical fiber was employed for distributed temperature sensing [258, 259]; however, most of the reported microfiber sensors are based on external Raman scattering induced by surrounding media [260, 261]. In these studies, the microfiber's large evanescent field was typically employed as the probe to interact with the surroundings. However, the efficiency of Raman scattering is extremely low. Thus, it typically requires a high-powered pump source and a highly 
sensitive spectrometer or a long microfiber to generate Raman signals with sufficient intensity. With the emergence of surface-enhanced Raman scattering, microfibers and microfiber tips decorated with plasmonic structures have been employed for ultra-sensitive molecular sensing [262-266].

The strong optical confinement and significant evanescent fields in microfiber are beneficial for ultra-sensitive molecular fluorescence spectroscopy [267-269]. In comparison with the absorption spectroscopy, the fluorescence spectroscopy contains two physical processes: fluorescence excitation and collection [270, 271]. Both processes can be implemented via evanescent coupling of the microfiber guided modes. Li et al. [269] reported a hybrid microfiber-microfluidic device for fluorescence measurements with a detection limit as low as $100 \mathrm{pM}$ and excellent reversibility in a concentration range between $0 \mathrm{nM}$ and $10 \mathrm{nM}$. In addition to embedding microfibers in the microchannels of microfluidic chips, hollow core microfiber has been employed for optofluidic manipulation and fluorescence detection in fluidics with an effective detection volume at the femtoliter scale [267] (Fig. 12). There is a growing interest in interfacing atoms with optical microfiber to manipulate and probe the atomic fluorescence [36, 272-274]. For example, spontaneous emission rates of excited atoms/molecules were investigated when positioned around a subwavelength microfiber, which opened a promising field in quantum electrodynamics [272, 274].

Moreover, microfiber tips have been used as fluorescence probes for bio/chemical detection. The nanoscale fiber tip is typically fabricated by cleaving a tapered fiber and functionalized using metal layer coated on the end face or functional materials (e.g., bioreceptors [275], dyes [276, 277], semiconductor/doped-polymer nanowires [278], and plasmonic structures [279]) attached to the tip. The micro-tip sensors exhibited excellent capabilities of single-cell-level investigations into chemical reactions in biosystems. Moreover, they provided minimally invasive tools to probe subcellular compartments inside individual living cells for health effect studies and medical applications [279].

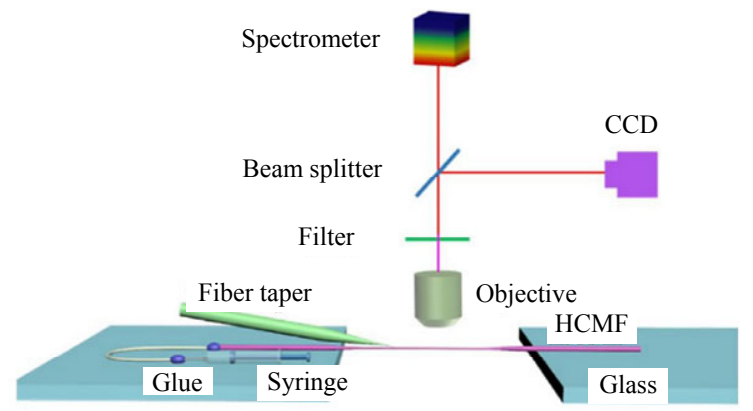

(a)

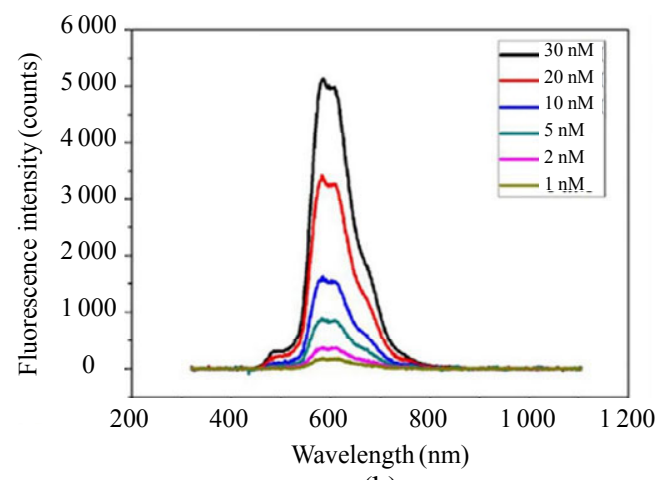

(b)

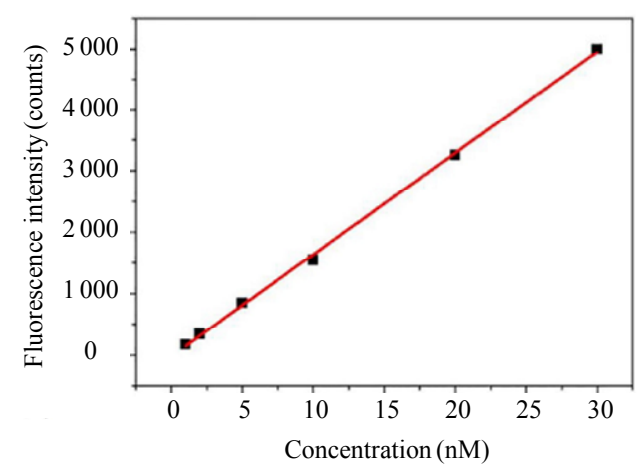

(c)

Fig. 12 Frequency-based microfiber fluorescence sensor: (a) scheme of the experimental setup for a hollow-core-microfiber-based fluorescence detector. Reproduced with permission [267]. Copyright 2018 Elsevier; (b) fluorescence spectra of fluorescent microsphere suspensions at different concentrations for the hollow-core-microfiber-based fluorescence detector. Reproduced with permission [267]. Copyright 2018 Elsevier; (c) fluorescence peak intensity as a function of concentration of fluorescent microsphere suspensions for the hollow-core-microfiber-based fluorescence detector. Reproduced with permission [267]. Copyright 2018 Elsevier. 


\section{Challenges and prospects}

Although significant progress has been made for microfiber-optic sensors in the past decades, we notice that several limitations must be resolved to enable wider applications. The first challenge is the fabrication of microfiber-based sensors with high repeatability, good scalability, and long-term stability. This is crucial for practical applications, especially for migrating microfiber sensors from laboratories to commercialized products. Thus far, a technique has been proposed to precisely manufacture microfibers with in-situ control of the waist diameter [280]. Packaging methods, including embedding the microfibers in low-RI materials [69, 70, 217, 281-283], integrating them with microfluidic chips [219, 269], and sealing them in glass tubes [284], were also demonstrated for better protection of sensitive microfiber sensing elements. However, the limitation regarding the efficient manipulation of fragile microfibers and performance maintenance after packaging still remains. The second challenge is the competition from other platforms, such as micro-electro-mechanical system sensors [285] and silicon photonic sensors [286, 287]. These integrated sensing devices rely on mature semiconductor fabrication techniques and offer the same advantages of a small footprint, high sensitivity, and fast response. Thus, it is critical to find the unique superiorities of microfiber sensors in some specific applications. Recently, with the emergence of the wearable technology, highly flexible microfiber-optic sensors may be utilized for human health monitoring and human-machine interaction [13, 288, 289].

\section{Conclusions}

We have reviewed the typical microfiber structures that can be harnessed for optical sensing. We have also summarized the implementations of microfiber-optic sensors based on different operating mechanisms, including interference, resonance, absorption, leakage, scattering, and fluorescence effects. We discussed the remaining challenges and concluded with prospects for future development of more practical microfiber-optic sensors. The microfiber platform will continue to offer increasing opportunities for sensing applications in combination with new structures and functional materials. It seems promising that the limitations of existing microfiber-optic sensors may be solved in the near future, and we expect that the sensors will be finally implemented in commercialized applications.

Open Access This article is distributed under the terms of the Creative Commons Attribution 4.0 International License (http://creativecommons.org/licenses/by/4.0/), which permits unrestricted use, distribution, and reproduction in any medium, provided you give appropriate credit to the original author(s) and the source, provide a link to the Creative Commons license, and indicate if changes were made.

\section{References}

[1] C. W. Hansell, "Picture transmission," U.S. Patent 1,751,584, Mar. 25, 1930.

[2] A. C. S. Van Heel, "A new method of transporting optical images without aberrations," Nature, 1954, 173(4392): 39.

[3] H. H. Hopkins and N. S. Kapany, "A flexible fibrescope, using static scanning," Nature, 1954, 173(4392): 39-41.

[4] E. Udd and W. B. Spillman, Fiber optic sensors: an introduction for engineers and scientists. Hoboken: John Wiley \& Sons, Inc., 2011.

[5] K. C. Kao and G. A. Hockham, "Dielectric-fibre surface waveguides for optical frequencies," Proceedings of the Institution of Electrical Engineers, 1966, 113(7): 1151-1158.

[6] F. P. Kapron, D. B. Keck, and R. D. Maurer, "Radiation losses in glass optical waveguides," Applied Physics Letters, 1970, 17(10): 423-425.

[7] R. Bergh, H. Lefevre, and H. Shaw, "An overview of fiber-optic gyroscopes," Journal of Lightwave Technology, 1984, 2(2): 91-107.

[8] J. A. Bucaro, H. D. Dardy, and E. F. Carome, "Fiber-optic hydrophone," The Journal of the Acoustical Society of America, 1977, 62(5): 1302-1304.

[9] T. Yoshino, K. Kurosawa, K. Itoh, and T. Ose, "Fiber-optic Fabry-Perot interferometer and its 
sensor applications," IEEE Transactions on Microwave Theory and Techniques, 1982, 30(10): 1612-1621.

[10] A. D. Kersey, M. A. Davis, H. J. Patrick, M. Leblanc, K. P. Koo, C. G. Askins, et al., "Fiber grating sensors," Journal of Lightwave Technology, 1997, 15(8): 1442-1463.

[11] B. Lee, "Review of the present status of optical fiber sensors," Optical Fiber Technology, 2003, 9(2): 57-79.

[12] M. F. S. Ferreira, E. Castro-Camus, D. J. Ottaway, J. M. López-Higuera, X. Feng, W. Jin, et al., "Roadmap on optical sensors," Journal of Optics, 2017, 19(8): 083001.

[13] L. Zhang, Y. Tang, and L. Tong, "Micro-/nanofiber optics: Merging photonics and material science on nanoscale for advanced sensing technology," Iscience, 2020, 23(1): 100810.

[14] G. Brambilla, F. Xu, P. Horak, Y. Jung, F. Koizumi, N. P. Sessions, E. Koukharenko, et al., "Optical fiber nanowires and microwires: Fabrication and applications," Advances in Optics and Photonics, 2009, 1(1): 107-161.

[15] R. Ismaeel, T. Lee, M. Ding, M. Belal, and G. Brambilla, "Optical microfiber passive components," Laser \& Photonics Reviews, 2013, 7(3): 350-384.

[16] L. Tong, "Micro/nanofibre optical sensors: Challenges and prospects," Sensors, 2018, 18(3): 903.

[17] L. Tong, F. Zi, X. Guo, and J. Lou, "Optical microfibers and nanofibers: A tutorial," Optics Communications, 2012, 285(23): 4641-4647.

[18] L. Tong, R. R. Gattass, J. B. Ashcom, S. He, J. Lou, M. Shen, et al., "Subwavelength-diameter silica wires for low-loss optical wave guiding," Nature, 2003, 426(6968): 816-819.

[19] L. Tong, J. Lou, and E. Mazur, "Single-mode guiding properties of subwavelength-diameter silica and silicon wire waveguides," Optics Express, 2004, 12(6): 1025-1035.

[20] J. Lou, L. Tong, and Z. Ye, "Modeling of silica nanowires for optical sensing," Optics Express, 2005, 13(6): 2135-2140.

[21] J. L. Kou, M. Ding, J. Feng, Y. Q. Lu, F. Xu, and G. Brambilla, "Microfiber-based Bragg gratings for sensing applications: a review," Sensors, 2012, 12(7): 8861-8876.

[22] S. C. Yan and F. Xu, "A review on optical microfibers in fluidic applications," Journal of Micromechanics and Microengineering, 2017, 27(9): 093001.

[23] J. C. Knight, G. Cheung, F. Jacques, and T. A. Birks, "Phase-matched excitation of whispering-gallerymode resonances by a fiber taper," Optics Letters, 1997, 22(15): 1129-1131.

[24] K. Huang, S. Yang, and L. Tong, "Modeling of evanescent coupling between two parallel optical nanowires," Applied Optics, 2007, 46(9): $1429-1434$

[25] F. Le Kien, V. I. Balykin, and K. Hakuta, “Atom trap and waveguide using a two-color evanescent light field around a subwavelength-diameter optical fiber," Physical Review A, 2004, 70(6): 063403.

[26] G. S. Murugan, G. Brambilla, J. S. Wilkinson, and D. J. Richardson, "Optical propulsion of individual and clustered microspheres along sub-micron optical wires," Japanese Journal of Applied Physics, 2008, 47(8S1): 6716-6718.

[27] J. Lægsgaard, "Theory of surface second-harmonic generation in silica nanowires," Journal of the Optical Society of America B, 2010, 27(7): 1317-1324.

[28] M. A. Gouveia, T. Lee, R. Ismaeel, M. Ding, N. G. R. Broderick, C. M. B. Cordeiro, et al., "Second harmonic generation and enhancement in microfibers and loop resonators," Applied Physics Letters, 2013, 102(20): 201120.

[29] A. Coillet and P. Grelu, "Third-harmonic generation in optical microfibers: From silica experiments to highly nonlinear glass prospects," Optics Communications, 2012, 285(16): 3493-3497.

[30] M. I. M. A. Khudus, T. Lee, F. De Lucia, C. Corbari, P. Sazio, P. Horak, et al., "All-fiber fourth and fifth harmonic generation from a single source," Optics Express, 2016, 24(19): 21777-21793.

[31] Y. Wang, T. Lee, F. De Lucia, M. I. M. Abdul Khudus, P. J. A. Sazio, M. Beresna, et al., "All-fiber sixth-harmonic generation of deep UV," Optics Letters, 2017, 42(22): 4671-4674.

[32] Y. H. Li, Y. Y. Zhao, and L. J. Wang, "Demonstration of almost octave-spanning cascaded four-wave mixing in optical microfibers," Optics Letters, 2012, 37(16): 3441-3443.

[33] S. Tang, Z. Wu, F. Xu, and Y. Lu, "Simulation of optical microfiber strain sensors based on four-wave mixing," IEEE Sensors Journal, 2016, 16(9): 3068-3074.

[34] S. G. Leon-Saval, T. A. Birks, W. J. Wadsworth, P. S. J. Russell, and M. W. Mason, "Supercontinuum generation in submicron fibre waveguides," Optics Express, 2004, 12(13): 2864-2869.

[35] F. Le Kien, S. D. Gupta, K. P. Nayak, and K. Hakuta, "Nanofiber-mediated radiative transfer between two distant atoms," Physical Review A, 2005, 72(6): 063815.

[36] K. P. Nayak, M. Sadgrove, R. Yalla, F. L. Kien, and K. Hakuta, "Nanofiber quantum photonics," Journal of Optics, 2018, 20(7): 073001.

[37] G. Y. Chen, D. G. Lancaster, and T. M. Monro, "Optical microfiber technology for current, temperature, acceleration, acoustic, humidity and ultraviolet light sensing," Sensors, 2018, 18(1): 72.

[38] J. H. Chen, D. R. Li, and F. Xu, "Optical microfiber 
sensors: Sensing mechanisms, and recent advances," Journal of Lightwave Technology, 2019, 37(11): 2577-2589.

[39] B. Guan and Y. Huang, "Interface sensitized optical microfiber biosensors," Journal of Lightwave Technology, 2019, 37(11): 2616-2622.

[40] W. Talataisong, R. Ismaeel, and G. Brambilla, "A review of microfiber-based temperature sensors," Sensors, 2018, 18(2): 461.

[41] P. Wang, L. Bo, Y. Semenova, G. Farrell, and G. Brambilla, "Optical microfibre based photonic components and their applications in label-free biosensing," Biosensors, 2015, 5(3): 471-499.

[42] Y. Wu, B. Yao, C. Yu, and Y. Rao, "Optical graphene gas sensors based on microfibers: a review," Sensors, 2018, 18(4): 941.

[43] D. I. Yeom, E. C. Mägi, M. R. E. Lamont, M. A. F. Roelens, L. Fu, and B. J. Eggleton, "Low-threshold supercontinuum generation in highly nonlinear chalcogenide nanowires," Optics Letters, 2008, 33(7): 660-662.

[44] G. Brambilla, F. Koizumi, X. Feng, and D. J. Richardson, "Compound-glass optical nanowires," Electronics Letters, 2005, 41(7): 400-402.

[45] O. Aktaş and M. Bayındır, "Tapered nanoscale chalcogenide fibers directly drawn from bulk glasses as optical couplers for high-index resonators," Applied Optics, 2017, 56(3): 385-390.

[46] S. A. Harfenist, S. D. Cambron, E. W. Nelson, S. M. Berry, A. W. Isham, M. M. Crain, et al., "Direct drawing of suspended filamentary micro- and nanostructures from liquid polymers," Nano Letters, 2004, 4(10): 1931-1937.

[47] X. Xing, Y. Wang, and B. Li, "Nanofiber drawing and nanodevice assembly in poly(trimethylene terephthalate)," Optics Express, 2008, 16(14): 10815-10822.

[48] F. Gu, H. Yu, P. Wang, Z. Yang, and L. Tong, "Light-emitting polymer single nanofibers via waveguiding excitation," ACS Nano, 2010, 4(9): 5332-5338.

[49] P. Wang, Y. Wang, and L. Tong, "Functionalized polymer nanofibers: A versatile platform for manipulating light at the nanoscale," Light: Science \& Applications, 2013, 2(10): e102.

[50] L. Persano, A. Camposeo, and D. Pisignano, "Active polymer nanofibers for photonics, electronics, energy generation and micromechanics," Progress in Polymer Science, 2015, 43: 48-95.

[51] G. Brambilla, V. Finazzi, and D. J. Richardson, "Ultra-low-loss optical fiber nanotapers," Optics Express, 2004, 12(10): 2258-2263.

[52] M. Sumetsky, Y. Dulashko, and A. Hale, "Fabrication and study of bent and coiled free silica nanowires: Self-coupling microloop optical interferometer," Optics Express, 2004, 12(15): $3521-3531$.
[53] E. J. Zhang, W. D. Sacher, and J. K. S. Poon, "Hydrofluoric acid flow etching of low-loss subwavelength-diameter biconical fiber tapers," Optics Express, 2010, 18(21): 22593-22598.

[54] L. Tong, L. Hu, J. Zhang, J. Qiu, Q. Yang, J. Lou, et al., "Photonic nanowires directly drawn from bulk glasses," Optics Express, 2006, 14(1): 82-87.

[55] R. Nagai and T. Aoki, "Ultra-low-loss tapered optical fibers with minimal lengths," Optics Express, 2014, 22(23): 28427-28436.

[56] L. Shi, X. Chen, H. Liu, Y. Chen, Z. Ye, W. Liao, et al., "Fabrication of submicron-diameter silica fibers using electric strip heater," Optics Express, 2006, 14(12): 5055-5060.

[57] I. Yokohama, J. Noda, and K. Okamoto, "Fiber-coupler fabrication with automatic fusion-elongation processes for low excess loss and high coupling-ratio accuracy," Journal of Lightwave Technology, 1987, 5(7): 910-915.

[58] F. Bilodeau, K. O. Hill, S. Faucher, and D. C. Johnson, "Low-loss highly overcoupled fused couplers: Fabrication and sensitivity to external pressure," Journal of Lightwave Technology, 1988, 6(10): 1476-1482.

[59] G. Brambilla and D. N. Payne, "The ultimate strength of glass silica nanowires," Nano Letters, 2009, 9(2): 831-835.

[60] L. Tong, J. Lou, R. R. Gattass, S. He, X. Chen, L. Liu, et al., "Assembly of silica nanowires on silica aerogels for microphotonic devices," Nano Letters, 2005, 5(2): 259-262.

[61] H. Yu, S. Wang, J. Fu, M. Qiu, Y. Li, F. Gu, et al. "Modeling bending losses of optical nanofibers or nanowires," Applied Optics, 2009, 48(22): 4365-4369.

[62] M. Sumetsky, Y. Dulashko, J. M. Fini, and A. Hale, "Optical microfiber loop resonator," Applied Physics Letters, 2005, 86(16): 161108.

[63] M. Sumetsky, Y. Dulashko, J. M. Fini, A. Hale, and D. J. Digiovanni, "The microfiber loop resonator: Theory, experiment, and application," Journal of Lightwave Technology, 2006, 24(1): 242-250.

[64] X. Jiang, Q. Yang, G. Vienne, Y. Li, L. Tong, J. Zhang, et al., "Demonstration of microfiber knot laser," Applied Physics Letters, 2006, 89(14): 143513.

[65] X. Jiang, Q. Song, L. Xu, J. Fu, and L. Tong, "Microfiber knot dye laser based on the evanescent-wave-coupled gain," Applied Physics Letters, 2007, 90(23): 233501.

[66] M. Sumetsky, "Optical fiber microcoil resonator," Optics Express, 2004, 12(10): 2303-2316.

[67] M. Sumetsky, "Uniform coil optical resonator and waveguide: transmission spectrum, eigenmodes, and dispersion relation," Optics Express, 2005, 13(11): 4331-4340.

[68] F. Xu, P. Horak, and G. Brambilla, "Optical 
microfiber coil resonator refractometric sensor," Optics Express, 2007, 15(12): 7888-7893.

[69] F. Xu and G. Brambilla, "Demonstration of a refractometric sensor based on optical microfiber coil resonator," Applied Physics Letters, 2008, 92(10): 101126.

[70] F. $\mathrm{Xu}$ and G. Brambilla, "Embedding optical microfiber coil resonators in Teflon," Optics Letters, 2007, 32(15): 2164-2166.

[71] P. Wang, M. Ding, G. Brambilla, Y. Semenova, Q. $\mathrm{Wu}$, and G. Farrell, "High temperature performance of an optical microfibre coupler and its potential use as a sensor," Electronics Letters, 2012, 48(5): 283-284.

[72] M. Ding, P. Wang, and G. Brambilla, "Fast-response high-temperature microfiber coupler tip thermometer," IEEE Photonics Technology Letters, 2012, 24(14): 1209-1211.

[73] B. S. Kawasaki, K. O. Hill, and R. G. Lamont, "Biconical-taper single-mode fiber coupler," Optics Letters, 1981, 6(7): 327-328.

[74] Y. Jung, G. Brambilla, and D. J. Richardson, "Optical microfiber coupler for broadband single-mode operation," Optics Express, 2009, 17(7): 5273-5278.

[75] M. Ding, P. Wang, and G. Brambilla, "A microfiber coupler tip thermometer," Optics Express, 2012, 20(5): 5402-5408.

[76] K. Liu, Y. He, A. Yang, L. Shi, L. Huang, P. Zhou, et al., "Resonant response and mode conversion of the microsphere coupled with a microfiber coupler," Optics Letters, 2019, 44(4): 879-882.

[77] Y. Li and L. Tong, "Mach-Zehnder interferometers assembled with optical microfibers or nanofibers," Optics Letters, 2008, 33(4): 303-305.

[78] J. Wo, G. Wang, Y. Cui, Q. Sun, R. Liang, P. P. Shum, et al., "Refractive index sensor using microfiber-based Mach-Zehnder interferometer," Optics Letters, 2012, 37(1): 67-69.

[79] J. Li, L. P. Sun, S. Gao, Z. Quan, Y. L. Chang, Y. Ran, et al., "Ultrasensitive refractive-index sensors based on rectangular silica microfibers," Optics Letters, 2011, 36(18): 3593-3595.

[80] L. Sun, J. Li, Y. Tan, X. Shen, X. Xie, S. Gao, et al., "Miniature highly-birefringent microfiber loop with extremely-high refractive index sensitivity," Optics Express, 2012, 20(9): 10180-10185.

[81] W. B. Ji, H. H. Liu, S. C. Tjin, K. K. Chow, and A. Lim, "Ultrahigh sensitivity refractive index sensor based on optical microfiber," IEEE Photonics Technology Letters, 2012, 24(20): 1872-1874.

[82] C. R. Biazoli, S. Silva, M. a. R. Franco, O. Frazão, and C. M. B. Cordeiro, "Multimode interference tapered fiber refractive index sensors," Applied Optics, 2012, 51(24): 5941-5945.

[83] C. Li, S. Qiu, Y. Chen, F. Xu, and Y. Lu, "Ultra-sensitive refractive index sensor with slightly tapered photonic crystal fiber," IEEE Photonics Technology Letters, 2012, 24(19): 1771-1774.

[84] J. Yang, L. Jiang, S. Wang, B. Li, M. Wang, H. Xiao, et al., "High sensitivity of taper-based Mach-Zehnder interferometer embedded in a thinned optical fiber for refractive index sensing," Applied Optics, 2011, 50(28): 5503-5507.

[85] L. Xu, Y. Li, and B. Li, "Nonadiabatic fiber taper-based Mach-Zehnder interferometer for refractive index sensing," Applied Physics Letters, 2012, 101(15): 153510 .

[86] M. I. Zibaii, H. Latifi, M. Karami, M. Gholami, S. M. Hosseini, and M. H. Ghezelayagh, "Non-adiabatic tapered optical fiber sensor for measuring the interaction between $\alpha$-amino acids in aqueous carbohydrate solution," Measurement Science and Technology, 2010, 21(10): 105801.

[87] L. P. Sun, J. Li, L. Jin, and B. O. Guan, "Structural microfiber long-period gratings," Optics Express, 2012, 20(16): 18079-18084.

[88] B. L. Li, J. H. Chen, F. Xu, and Y. Q. Lu, "Periodic micro-structures in optical microfibers induced by Plateau-Rayleigh instability and its applications," Optics Express, 2017, 25(4): 4326-4334.

[89] Y. Tan, L. P. Sun, L. Jin, J. Li, and B. O. Guan, "Microfiber Mach-Zehnder interferometer based on long period grating for sensing applications," Optics Express, 2013, 21(1): 154-164.

[90] B. S. Kawasaki and K. O. Hill, "Low-loss access coupler for multimode optical fiber distribution networks," Applied Optics, 1977, 16(7): 1794-1795.

[91] R. A. Bergh, G. Kotler, and H. J. Shaw, "Single-mode fibre optic directional coupler," Electronics Letters, 1980, 16(7): 260-261.

[92] Y. Szu-Wen, W. Tzong-Lin, W. Cheng Wen, and C. Hung-Chun, "Numerical modeling of weakly fused fiber-optic polarization beamsplitters. Part II: the three-dimensional electromagnetic model," Journal of Lightwave Technology, 1998, 16(4): 691.

[93] C. R. Liao, D. N. Wang, X. He, and M. W. Yang, "Twisted optical microfibers for refractive index sensing," IEEE Photonics Technology Letters, 2011, 23(13): 848-850.

[94] S. C. Yan, Y. Chen, C. Li, F. Xu, and Y. Q. Lu, "Differential twin receiving fiber-optic magnetic field and electric current sensor utilizing a microfiber coupler," Optics Express, 2015, 23(7): 9407-9414.

[95] Q. Zhang, J. Lei, B. Cheng, Y. Song, L. Hua, and H. Xiao, "A microfiber half coupler for refractive index sensing," IEEE Photonics Technology Letters, 2017, 29(18): 1525-1528.

[96] S. Pu, L. Luo, J. Tang, L. Mao, and X. Zeng, "Ultrasensitive refractive-index sensors based on tapered fiber coupler with Sagnac loop," IEEE Photonics Technology Letters, 2016, 28(10): 1073-1076. 
[97] Y. Chen, S. C. Yan, X. Zheng, F. Xu, and Y. Q. Lu, "A miniature reflective micro-force sensor based on a microfiber coupler," Optics Express, 2014, 22(3): 2443-2450.

[98] L. Zu, H. Zhang, Y. Miao, and B. Li, "Microfiber coupler with a Sagnac loop for water pollution detection," in Proceedings of 18th International Conference on Optical Communications and Networks (ICOCN), Anhui, 2019, pp. 1-3.

[99] H. Luo, Q. Sun, X. Li, Z. Yan, Y. Li, D. Liu, et al., "Refractive index sensitivity characteristics near the dispersion turning point of the multimode microfiber-based Mach-Zehnder interferometer," Optics Letters, 2015, 40(21): 5042-5045.

[100] K. Li, T. Zhang, G. Liu, N. Zhang, M. Zhang, and L. Wei, "Ultrasensitive optical microfiber coupler based sensors operating near the turning point of effective group index difference," Applied Physics Letters, 2016, 109(10): 101101.

[101] D. A. Jackson, A. Dandridge, and S. K. Sheem, "Measurement of small phase shifts using a single-mode optical-fiber interferometer," Optics Letters, 1980, 5(4): 139-141.

[102] R. Jha, J. Villatoro, G. Badenes, and V. Pruneri, "Refractometry based on a photonic crystal fiber interferometer," Optics Letters, 2009, 34(5): 617-619.

[103] Y. Du, Y. Chen, Y. Zhuang, C. Zhu, F. Tang, and J. Huang, "Probing nanostrain via a mechanically designed optical fiber interferometer," IEEE Photonics Technology Letters, 2017, 29(16): 1348-1351.

[104] Y. Xue, Y. S. Yu, R. Yang, C. Wang, C. Chen, J. C. Guo, et al., "Ultrasensitive temperature sensor based on an isopropanol-sealed optical microfiber taper," Optics Letters, 2013, 38(8): 1209-1211.

[105] M. F. Jaddoa, A. A. Jasim, M. Z. A. Razak, S. W. Harun, and $\mathrm{H}$. Ahmad, "Highly responsive $\mathrm{NaCl}$ detector based on inline microfiber Mach-Zehnder interferometer," Sensors and Actuators A: Physical, 2016, 237: 56-61.

[106] V. P. Minkovich, J. Villatoro, D. Monzón-Hernández, S. Calixto, A. B. Sotsky, and L. I. Sotskaya, "Holey fiber tapers with resonance transmission for high-resolution refractive index sensing," Optics Express, 2005, 13(19): 7609-7614.

[107] P. Wang, M. Ding, L. Bo, C. Guan, Y. Semenova, W. Sun, et al., "Photonic crystal fiber half-taper probe based refractometer," Optics Letters, 2014, 39(7): 2076-2079.

[108] S. J. Qiu, Y. Chen, J. L. Kou, F. Xu, and Y. Q. Lu, "Miniature tapered photonic crystal fiber interferometer with enhanced sensitivity by acid microdroplets etching," Applied Optics, 2011, 50(22): 4328-4332.

[109] P. Wang, M. Ding, L. Bo, C. Guan, Y. Semenova, Q.
Wu, et al., "Fiber-tip high-temperature sensor based on multimode interference," Optics Letters, 2013, 38(22): 4617-4620.

[110] R. M. Andre, C. R. Biazoli, S. O. Silva, M. B. Marques, C. M. B. Cordeiro, and O. Frazao, "Strain-temperature discrimination using multimode interference in tapered fiber," IEEE Photonics Technology Letters, 2013, 25(2): $155-158$.

[111] T. Erdogan, "Fiber grating spectra," Journal of Lightwave Technology, 1997, 15(8): 1277-1294.

[112] K. O. Hill, Y. Fujii, D. C. Johnson, and B. S. Kawasaki, "Photosensitivity in optical fiber waveguides: Application to reflection filter fabrication," Applied Physics Letters, 1978, 32(10): 647-649.

[113] K. O. Hill and G. Meltz, "Fiber Bragg grating technology fundamentals and overview," Journal of Lightwave Technology, 1997, 15(8): 1263-1276.

[114] B. O. Guan, J. Li, L. Jin, and Y. Ran, "Fiber Bragg gratings in optical microfibers," Optical Fiber Technology, 2013, 19(6): 793-801.

[115] A. Iadicicco, A. Cusano, A. Cutolo, R. Bernini, and M. Giordano, "Thinned fiber Bragg gratings as high sensitivity refractive index sensor," IEEE Photonics Technology Letters, 2004, 16(4): 1149-1151.

[116] W. Liang, Y. Huang, Y. Xu, R. K. Lee, and A. Yariv, "Highly sensitive fiber Bragg grating refractive index sensors," Applied Physics Letters, 2005, 86(15): 151122 .

[117] G. Kakarantzas, T. E. Dimmick, T. A. Birks, R. L. Roux, and P. S. J. Russell, "Miniature all-fiber devices based on $\mathrm{CO}_{2}$ laser microstructuring of tapered fibers," Optics Letters, 2001, 26(15): 1137-1139.

[118] X. Fang, C. R. Liao, and D. N. Wang, "Femtosecond laser fabricated fiber Bragg grating in microfiber for refractive index sensing," Optics Letters, 2010, 35(7): 1007-1009.

[119] Y. Zhang, B. Lin, S. C. Tjin, H. Zhang, G. Wang, P. Shum, et al., "Refractive index sensing based on higher-order mode reflection of a microfiber Bragg grating," Optics Express, 2010, 18(25): 26345-26350.

[120] P. Zhao, Y. Li, J. Zhang, L. Shi, and X. Zhang, "Nanohole induced microfiber Bragg gratings," Optics Express, 2012, 20(27): 28625-28630.

[121] C. Liao, K. Yang, J. Wang, Z. Bai, Z. Gan, and Y. Wang, "Helical microfiber Bragg grating printed by femtosecond laser for refractive index sensing," IEEE Photonics Technology Letters, 2019, 31(12): 971-974.

[122] K. P. Nayak, F. Le Kien, Y. Kawai, K. Hakuta, K. Nakajima, H. T. Miyazaki, et al., "Cavity formation on an optical nanofiber using focused ion beam milling technique," Optics Express, 2011, 19(15): 
14040-14050.

[123] Y. Liu, C. Meng, A. P. Zhang, Y. Xiao, H. Yu, and L. Tong, "Compact microfiber Bragg gratings with high-index contrast," Optics Letters, 2011, 36(16): 3115-3117.

[124] J. L. Kou, S. J. Qiu, F. Xu, and Y. Q. Lu, "Demonstration of a compact temperature sensor based on first-order Bragg grating in a tapered fiber probe," Optics Express, 2011, 19(19): 18452-18457.

[125] M. Ding, P. Wang, T. Lee, and G. Brambilla, "A microfiber cavity with minimal-volume confinement," Applied Physics Letters, 2011, 99(5): 051105 .

[126] M. Ding, M. N. Zervas, and G. Brambilla, "A compact broadband microfiber Bragg grating," Optics Express, 2011, 19(16): 15621-15626.

[127] J. Feng, M. Ding, J. Kou, F. Xu, and Y. Lu, "An optical fiber tip micrograting thermometer," IEEE Photonics Journal, 2011, 3(5): 810-814.

[128] J. Kou, S. Qiu, F. Xu, Y. Lu, Y. Yuan, and G. Zhao, "Miniaturized metal-dielectric-hybrid fiber tip grating for refractive index sensing," IEEE Photonics Technology Letters, 2011, 23(22): $1712-1714$

[129] W. Ding, S. R. Andrews, T. A. Birks, and S. A. Maier, "Modal coupling in fiber tapers decorated with metallic surface gratings," Optics Letters, 2006, 31(17): 2556-2558.

[130] Y. Shen, L. Yao, Z. Li, J. Kou, Y. Cui, J. Bian, et al., "Double transfer UV-curing nanoimprint lithography," Nanotechnology, 2013, 24(46): 465304.

[131] F. Xu, G. Brambilla, and Y. Lu, "A microfluidic refractometric sensor based on gratings in optical fibre microwires," Optics Express, 2009, 17(23): 20866-20871.

[132] F. Xu, G. Brambilla, J. Feng, and Y. Lu, "A microfiber Bragg grating based on a microstructured rod: a proposal," IEEE Photonics Technology Letters, 2010, 22(4): 218-220.

[133] J. L. Kou, Z. D. Huang, G. Zhu, F. Xu, and Y. Q. Lu, "Wave guiding properties and sensitivity of D-shaped optical fiber microwire devices," Applied Physics B, 2011, 102(3): 615-619.

[134] K. P. Nayak and K. Hakuta, "Photonic crystal formation on optical nanofibers using femtosecond laser ablation technique," Optics Express, 2013, 21(2): 2480-2490.

[135] H. Takashima, A. Fukuda, H. Maruya, T. Tashima, A. W. Schell, and S. Takeuchi, "Fabrication of a nanofiber Bragg cavity with high quality factor using a focused helium ion beam," Optics Express, 2019, 27(5): 6792-6800.

[136] R. Yalla, M. Sadgrove, K. P. Nayak, and K. Hakuta, "Cavity quantum electrodynamics on a nanofiber using a composite photonic crystal cavity,"
Physical Review Letters, 2014, 113(14): 143601.

[137] J. Kou, F. Xu, and H. Choo, "Implementation of a high-Q, small mode volume cavity in microfibers using lattice-constant-varying nanohole arrays," IEEE Journal of Selected Topics in Quantum Electronics, 2014, 20(5): 85-88.

[138] I. M. White, H. Oveys, and X. Fan, "Liquid-core optical ring-resonator sensors," Optics Letters, 2006, 31(9): 1319-1321.

[139] M. Sumetsky, R. S. Windeler, Y. Dulashko, and X. Fan, "Optical liquid ring resonator sensor," Optics Express, 2007, 15(22): 14376-14381.

[140] J. L. Kou, J. Feng, Q. J. Wang, F. Xu, and Y. Q. Lu, "Microfiber-probe-based ultrasmall interferometric sensor," Optics Letters, 2010, 35(13): 2308-2310.

[141] J. L. Kou, J. Feng, L. Ye, F. Xu, and Y. Q. Lu, "Miniaturized fiber taper reflective interferometer for high temperature measurement," Optics Express, 2010, 18(13): 14245-14250.

[142] S. S. Wang, Z. F. Hu, Y. H. Li, and L. M. Tong, "All-fiber Fabry-Perot resonators based on microfiber sagnac loop mirrors," Optics Letters, 2009, 34(3): 253-255.

[143] G. Vienne, A. Coillet, P. Grelu, M. E. Amraoui, J. C. Jules, F. Smektala, et al., "Demonstration of a reef knot microfiber resonator," Optics Express, 2009, 17(8): 6224-6229.

[144] Y. Jung, G. Brambilla, G. S. Murugan, and D. J. Richardson, "Optical racetrack ring-resonator based on two U-bent microfibers," Applied Physics Letters, 2011, 98(2): 021109.

[145] R. Ismaeel, T. Lee, F. Al-Saab, Y. Jung, and G. Brambilla, "A self-coupling multi-port microcoil resonator," Optics Express, 2012, 20(8): 8568-8574.

[146] Z. Xu, Q. Sun, B. Li, Y. Luo, W. Lu, D. Liu, et al., "Highly sensitive refractive index sensor based on cascaded microfiber knots with Vernier effect," Optics Express, 2015, 23(5): 6662-6672.

[147] Z. Xu, Y. Luo, D. Liu, P. P. Shum, and Q. Sun, "Sensitivity-controllable refractive index sensor based on reflective $\theta$-shaped microfiber resonator cooperated with Vernier effect," Scientific Reports, 2017, 7(1): 9620.

[148] C. Y. Chao and L. J. Guo, "Design and optimization of microring resonators in biochemical sensing applications," Journal of Lightwave Technology, 2006, 24(3): 1395-1402.

[149] I. M. White and X. Fan, "On the performance quantification of resonant refractive index sensors," Optics Express, 2008, 16(2): 1020-1028.

[150] X. Guo, M. Qiu, J. Bao, B. J. Wiley, Q. Yang, X. Zhang, et al., "Direct coupling of plasmonic and photonic nanowires for hybrid nanophotonic components and circuits," Nano Letters, 2009, 9(12): 4515-4519.

[151] S. C. Yan, Z. Y. Liu, C. Li, S. J. Ge, F. Xu, and Y. Q. 
Lu, "Hot-wire" microfluidic flowmeter based on a microfiber coupler," Optics Letters, 2016, 41(24): 5680-5683.

[152] F. Gu, G. Wu, and H. Zeng, "Hybrid photon-plasmon Mach-Zehnder interferometers for highly sensitive hydrogen sensing," Nanoscale, 2015, 7(3): 924-929.

[153] J. H. Chen, Y. Chen, W. Luo, J. L. Kou, F. Xu, and Y. Q. Lu, "Multifunctional optical nanofiber polarization devices with 3D geometry," Optics Express, 2014, 22(15): 17890-17896.

[154] H. Y. Lin, C. H. Huang, G. L. Cheng, N. K. Chen, and H. C. Chui, "Tapered optical fiber sensor based on localized surface plasmon resonance," Optics Express, 2012, 20(19): 21693-21701.

[155] Z. X. Ding, Z. N. Huang, Y. Chen, C. Mou, Y. Q. $\mathrm{Lu}$, and $\mathrm{F} . \mathrm{Xu}$, "All-fiber ultrafast laser generating gigahertz-rate pulses based on a hybrid plasmonic microfiber resonator," Advanced Photonics, 2020, 2(2): 026002.

[156] J. H. Li, J. H. Chen, S. C. Yan, Y. P. Ruan, F. Xu, and Y. Q. Lu, "Versatile hybrid plasmonic microfiber knot resonator," Optics Letters, 2017, 42(17): 3395-3398.

[157] D. Cai, T. Tong, Z. Zhang, J. Pan, L. Zhang, and L. Tong, "Functional film coated optical micro/nanofibers for high-performance gas sensing," IEEE Sensors Journal, 2019, 19(20): 9229-9234.

[158] D. Li, G. Wu, J. Chen, S. Yan, Z. Liu, F. Xu, et al., "Ethanol gas sensor based on a hybrid polymethyl methacrylate-silica microfiber coupler," Journal of Lightwave Technology, 2018, 36(10): 2031-2036.

[159] F. Gu, X. Yin, H. Yu, P. Wang, and L. Tong, "Polyaniline/polystyrene single-nanowire devices for highly selective optical detection of gas mixtures," Optics Express, 2009, 17(13): 11230-11235.

[160] F. Gu, L. Zhang, X. Yin, and L. Tong, "Polymer single-nanowire optical sensors," Nano Letters, 2008, 8(9): 2757-2761.

[161] J. H. Chen, G. Q. Deng, S. C. Yan, C. Li, K. Xi, F. $\mathrm{Xu}$, et al. "Microfiber-coupler-assisted control of wavelength tuning for Q-switched fiber laser with few-layer molybdenum disulfide nanoplates," Optics Letters, 2015, 40(15): 3576-3579.

[162] Y. Wu, B. Yao, A. Zhang, Y. Rao, Z. Wang, Y. Cheng, et al., "Graphene-coated microfiber bragg grating for high-sensitivity gas sensing," Optics Letters, 2014, 39(5): 1235-1237.

[163] S. Sridevi, K. S. Vasu, S. Sampath, S. Asokan, and A. K. Sood, "Optical detection of glucose and glycated hemoglobin using etched fiber Bragg gratings coated with functionalized reduced graphene oxide," Journal of Biophotonics, 2016, 9(7): 760-769.

[164] C. B. Yu, Y. Wu, X. L. Liu, B. C. Yao, F. Fu, Y.
Gong, et al., "Graphene oxide deposited microfiber knot resonator for gas sensing," Optical Materials Express, 2016, 6(3): 727-733.

[165] Y. Huang, B. Yu, T. Guo, and B. O. Guan, "Ultrasensitive and in situ DNA detection in various $\mathrm{pH}$ environments based on a microfiber with a graphene oxide linking layer," RSC Advances, 2017, 7(22): 13177-13183.

[166] B. C. Yao, Y. Wu, A. Q. Zhang, Y. J. Rao, Z. G. Wang, Y. Cheng, et al., "Graphene enhanced evanescent field in microfiber multimode interferometer for highly sensitive gas sensing," Optics Express, 2014, 22(23): 28154-28162.

[167] Y. Wu, B. C. Yao, A. Q. Zhang, X. L. Cao, Z. G. Wang, Y. J. Rao, et al., "Graphene-based D-shaped fiber multicore mode interferometer for chemical gas sensing," Optics Letters, 2014, 39(20): 6030-6033.

[168] J. Zhang, H. Fu, J. Ding, M. Zhang, and Y. Zhu, "Graphene-oxide-coated interferometric optical microfiber ethanol vapor sensor," Applied Optics, 2017, 56(31): 8828-8831.

[169] Y. Bai, Y. Miao, H. Zhang, and J. Yao, "Simultaneous measurement of relative humidity and temperature using a microfiber coupler coated with molybdenum disulfide nanosheets," Optical Materials Express, 2019, 9(7): 2846-2858.

[170] J. H. Chen, J. Tan, G. X. Wu, X. J. Zhang, F. Xu, and Y. Q. Lu, "Tunable and enhanced light emission in hybrid $\mathrm{WS}_{2}$-optical-fiber-nanowire structures," Light: Science \& Applications, 2019, 8(1): 1-8.

[171] D. Zhang, H. Guan, W. Zhu, J. Yu, H. Lu, W. Qiu, et al., "All light-control-light properties of molybdenum diselenide $\left(\mathrm{MoSe}_{2}\right)$-coatedmicrofiber," Optics Express, 2017, 25(23): 28536-28546.

[172] S. R. Azzuhri, I. S. Amiri, A. S. Zulkhairi, M. A. M. Salim, M. Z. A. Razak, M. F. Khyasudeen, et al., "Application of graphene oxide based microfiber-knot resonator for relative humidity sensing," Results in Physics, 2018, 9: 1572-1577.

[173] W. Li, B. Chen, C. Meng, W. Fang, Y. Xiao, X. Li, et al., "Ultrafast all-optical graphene modulator," Nano Letters, 2014, 14(2): 955-959.

[174] L. Sun, Y. Semenova, Q. Wu, D. Liu, J. Yuan, X. Sang, et al., "Investigation of humidity and temperature response of a silica gel coated microfiber coupler," IEEE Photonics Journal, 2016, 8(6): 1-7.

[175] H. Guo, F. Pang, X. Zeng, N. Chen, Z. Chen, and T. Wang, "Temperature sensor using an optical fiber coupler with a thin film," Applied Optics, 2008, 47(19): 3530-3534.

[176] L. Luo, S. Pu, J. Tang, X. Zeng, and M. Lahoubi, "Highly sensitive magnetic field sensor based on microfiber coupler with magnetic fluid," Applied 
Physics Letters, 2015, 106(19): 193507.

[177] Y. Zheng, X. Dong, C. C. Chan, P. P. Shum, and H. $\mathrm{Su}$, "Optical fiber magnetic field sensor based on magnetic fluid and microfiber mode interferometer," Optics Communications, 2015, 336: $5-8$.

[178] X. Li and H. Ding, "All-fiber magnetic-field sensor based on microfiber knot resonator and magnetic fluid," Optics Letters, 2012, 37(24): 5187-5189.

[179] T. Tan, X. Jiang, C. Wang, B. Yao, and H. Zhang, "2D material optoelectronics for information functional device applications: status and challenges," Advanced Science, 2020, 7: 2000058.

[180] W. Yang, L. Gan, H. Li, and T. Zhai, "Two-dimensional layered nanomaterials for gas-sensing applications," Inorganic Chemistry Frontiers, 2016, 3(4): 433-451.

[181] F. Bonaccorso, Z. Sun, T. Hasan, and A. C. Ferrari, "Graphene photonics and optoelectronics," Nature Photonics, 2010, 4(9): 611-622.

[182] K. Li, N. Zhang, N. M. Y. Zhang, W. Zhou, T. Zhang, M. Chen, et al., "Birefringence induced Vernier effect in optical fiber modal interferometers for enhanced sensing," Sensors and Actuators B: Chemical, 2018, 275: 16-24.

[183] Y. Jiang, Z. Fang, Y. Du, E. Lewis, G. Farrell, and P. Wang, "Highly sensitive temperature sensor using packaged optical microfiber coupler filled with liquids," Optics Express, 2018, 26(1): 356-366.

[184] L. Zhao, Y. Zhang, J. Wang, and Y. Chen, "Highly sensitive temperature sensor based on an isopropanol-sealed optical microfiber coupler," Applied Physics Letters, 2018, 113(11): 111901.

[185] Y. Peng, Y. Zhao, X. G. Hu, and M. Q. Chen, "Humidity sensor based on unsymmetrical U-shaped twisted microfiber coupler with wide detection range," Sensors and Actuators B: Chemical, 2019, 290: 406-413.

[186] Y. Zhao, Y. Peng, M. Q. Chen, F. Xia, and R. J. Tong, "U-shaped microfiber coupler coated with polyvinyl alcohol film for highly sensitive humidity detection," Sensors and Actuators A: Physical, 2019, 285: 628-636.

[187] M. V. Hernández-Arriaga, M. A. Bello-Jiménez, A. Rodríguez-Cobos, R. López-Estopier, and M. V. Andrés, "High sensitivity refractive index sensor based on highly overcoupled tapered fiber-optic couplers," IEEE Sensors Journal, 2017, 17(2): 333-339.

[188] K. T. Kim, K. J. Cho, K. Im, S. Baik, C. Lee, and J. Lee, "High sensitivity refractive index sensor based on a wet-etched fused fiber coupler," IEEE Sensors Journal, 2010, 11(7): 1568-1572.

[189] N. M. Y. Zhang, K. Li, N. Zhang, Y. Zheng, T. Zhang, M. Qi, et al., "Highly sensitive gas refractometers based on optical microfiber modal interferometers operating at dispersion turning point," Optics Express, 2018, 26(22): 29148-29158.

[190] J. Wang, Q. Sun, Y. Li, S. Tan, L. Yang, F. Fang, et al., "Highly sensitive liquid-level sensor based on an optical reflective microfiber probe," Optics Letters, 2020, 45(1): 169-172.

[191] B. Yao, Y. Wu, Y. Cheng, A. Zhang, Y. Gong, Y. J. Rao, et al., "All-optical Mach-Zehnder interferometric $\mathrm{NH}_{3}$ gas sensor based on graphene/microfiber hybrid waveguide," Sensors and Actuators B: Chemical, 2014, 194: 142-148.

[192] H. Luo, Q. Sun, Z. Xu, D. Liu, and L. Zhang, "Simultaneous measurement of refractive index and temperature using multimode microfiber-based dual Mach-Zehnder interferometer," Optics Letters, 2014, 39(13): 4049-4052.

[193] H. Luo, Q. Sun, Z. Xu, W. Jia, D. Liu, and L. Zhang, "Microfiber-based inline Mach-Zehnder interferometer for dual-parameter measurement," IEEE Photonics Journal, 2015, 7(2): 1-8.

[194] Q. Sun, H. Luo, H. Luo, M. Lai, D. Liu, and L. Zhang, "Multimode microfiber interferometer for dual-parameters sensing assisted by Fresnel reflection," Optics Express, 2015, 23(10): 12777-12783.

[195] Y. Li, H. Ma, L. Gan, Q. Liu, Z. Yan, D. Liu, et al., "Immobilized optical fiber microprobe for selective and high sensitive glucose detection," Sensors and Actuators B: Chemical, 2018, 255: 3004-3010.

[196] S. Lee, S. S. Saini, and M. Jeong, "Simultaneous measurement of refractive index, temperature, and strain using etched-core fiber Bragg grating sensors," IEEE Photonics Technology Letters, 2010, 22(19): 1431-1433.

[197] T. Wieduwilt, S. Brückner, and H. Bartelt, "High force measurement sensitivity with fiber Bragg gratings fabricated in uniform-waist fiber tapers," Measurement Science and Technology, 2011, 22(7): 075201.

[198] W. Luo, J. L. Kou, Y. Chen, F. Xu, and Y. Q. Lu, "Ultra-highly sensitive surface-corrugated microfiber Bragg grating force sensor," Applied Physics Letters, 2012, 101(13): 133502.

[199] M. Tian, Y. Huang, C. Li, and M. Lv, "High-performance humidity sensor based on a micro-nano fiber Bragg grating coated with graphene oxide," Optics Express, 2020, 28(18): 26395-26406.

[200] S. M. Lee, M. Y. Jeong, and S. S. Saini, "Etched-core fiber Bragg grating sensors integrated with microfluidic channels," Journal of Lightwave Technology, 2011, 30(8): 1025-1031.

[201] G. Kakarantzas, S. G. Leon-Saval, T. A. Birks, and P. S. J. Russell, "Low-loss deposition of solgel-derived silica films on tapered fibers," Optics Letters, 2004, 29(7): 694-696.

[202] H. Xuan, W. Jin, and M. Zhang, " $\mathrm{CO}_{2}$ laser induced 
long period gratings in optical microfibers," Optics Express, 2009, 17(24): 21882-21890.

[203] P. Fan, L. P. Sun, Z. Yu, J. Li, C. Wu, and B. O. Guan, "Higher-order diffraction of long-period microfiber gratings realized by arc discharge method," Optics Express, 2016, 24(22): 25380-25388.

[204] X. Li and H. Ding, "A stable evanescent field-based microfiber knot resonator refractive index sensor," IEEE Photonics Technology Letters, 2014, 26(16): 1625-1628.

[205] Y. Wu, Y. J. Rao, Y. H. Chen, and Y. Gong, "Miniature fiber-optic temperature sensors based on silica/polymer microfiber knot resonators," Optics Express, 2009, 17(20): 18142-18147.

[206] X. Zeng, Y. Wu, C. Hou, J. Bai, and G. Yang, “A temperature sensor based on optical microfiber knot resonator," Optics Communications, 2009, 282(18): 3817-3819.

[207] Z. Liu, X. Qiao, and R. Wang, "Miniaturized fiber-taper-based Fabry-Perot interferometer for high-temperature sensing," Applied Optics, 2017, 56(2): 256-259.

[208] Y. Wu, L. Jia, T. Zhang, Y. Rao, and Y. Gong, "Microscopic multi-point temperature sensing based on microfiber double-knot resonators," Optics Communications, 2012, 285(8): 2218-2222.

[209] J. Li, L. Gai, H. Li, and H. Hu, "A high sensitivity temperature sensor based on packaged microfibre knot resonator," Sensors and Actuators A: Physical, 2017, 263: 369-372.

[210] X. Guo, Y. Li, X. Jiang, and L. Tong, "Demonstration of critical coupling in microfiber loops wrapped around a copper rod," Applied Physics Letters, 2007, 91(7): 073512.

[211] S. C. Yan, B. C. Zheng, J. H. Chen, F. Xu, and Y. Q. $\mathrm{Lu}$, "Optical electrical current sensor utilizing a graphene-microfiber-integrated coil resonator," Applied Physics Letters, 2015, 107(5): 053502.

[212] J. Hou, H. Ding, B. Wei, C. Gao, and X. Li, "Microfiber knot resonator based electric field sensor," Instrumentation Science \& Technology, 2017, 45(3): 259-267.

[213] Y. Wu, T. Zhang, Y. Rao, and Y. Gong, "Miniature interferometric humidity sensors based on silica/polymer microfiber knot resonators," Sensors and Actuators B: Chemical, 2011, 155(1): 258-263.

[214] Y. Yin, S. Li, S. Wang, S. Jia, J. Ren, G. Farrell, et al., "Ultra-high-resolution detection of $\mathrm{Pb}^{2+}$ ions using a black phosphorus functionalized microfiber coil resonator," Photonics Research, 2019, 7(6): 622-629.

[215] Y. Yin, S. Li, J. Ren, G. Farrell, E. Lewis, and P. Wang, "High-sensitivity salinity sensor based on optical microfiber coil resonator," Optics Express, 2018, 26(26): 34633-34640.

[216] Y. Jung, G. S. Murugan, G. Brambilla, and D. J.
Richardson, "Embedded optical microfiber coil resonator with enhanced high-Q," IEEE Photonics Technology Letters, 2010, 22(22): 1638-1640.

[217] G. Vienne, Y. Li, and L. Tong, "Effect of host polymer on microfiber resonator," IEEE Photonics Technology Letters, 2007, 19(18): 1386-1388.

[218] F. Warken, E. Vetsch, D. Meschede, M. Sokolowski, and A. Rauschenbeutel, "Ultra-sensitive surface absorption spectroscopy using sub-wavelength diameter optical fibers," Optics Express, 2007, 15(19): 11952-11958.

[219] L. Zhang, P. Wang, Y. Xiao, H. Yu, and L. Tong, "Ultra-sensitive microfibre absorption detection in a microfluidic chip," Lab on a Chip, 2011, 11(21): 3720-3724.

[220] R. Lorenzi, Y. Jung, and G. Brambilla, "In-line absorption sensor based on coiled optical microfiber," Applied Physics Letters, 2011, 98(17): 173504.

[221] H. Waechter, J. Litman, A. H. Cheung, J. A. Barnes, and H. P. Loock, "Chemical sensing using fiber cavity ring-down spectroscopy," Sensors, 2010, 10(3): 1716-1742.

[222] J. Villatoro and D. Monzón-Hernández, "Fast detection of hydrogen with nano fiber tapers coated with ultra thin palladium layers," Optics Express, 2005, 13(13): 5087-5092.

[223] T. Wieduwilt, M. Zeisberger, M. Thiele, B. Doherty, M. Chemnitz, A. Csaki, et al., "Gold-reinforced silver nanoprisms on optical fiber tapers - a new base for high precision sensing," APL Photonics, 2016, 1(6): 066102.

[224] K. Li, G. Liu, Y. Wu, P. Hao, W. Zhou, and Z. Zhang, "Gold nanoparticle amplified optical microfiber evanescent wave absorption biosensor for cancer biomarker detection in serum," Talanta, 2014, 120: 419-424.

[225] G. Liu and K. Li, "Micro/nano optical fibers for label-free detection of abrin with high sensitivity," Sensors and Actuators B: Chemical, 2015, 215: 146-151.

[226] L. Zhang, F. Gu, J. Lou, X. Yin, and L. Tong, "Fast detection of humidity with a subwavelength-diameter fiber taper coated with gelatin film," Optics Express, 2008, 16(17): 13349-13353.

[227] P. C. A. Jerónimo, A. N. Araújo, and M. C. B. S. M. Montenegro, "Optical sensors and biosensors based on sol-gel films," Talanta, 2007, 72(1): 13-27.

[228] Y. Wu, B. Yao, Y. Cheng, Y. Rao, Y. Gong, W. Zhang, et al., "Hybrid graphene-microfiber waveguide for chemical gas sensing," IEEE Journal of Selected Topics in Quantum Electronics, 2014, 20(1): 49-54.

[229] H. Qiu, S. Gao, P. Chen, Z. Li, X. Liu, C. Zhang, et al., "Evanescent wave absorption sensor based on tapered multimode fiber coated with monolayer 
graphene film," Optics Communications, 2016, 366: 275-281.

[230] S. H. Girei, A. A. Shabaneh, H. Ngee-Lim, M. N. Hamidon, M. A. Mahdi, and M. H. Yaacob, "Tapered optical fiber coated with graphene based nanomaterials for measurement of ethanol concentrations in water," Optical Review, 2015, 22(3): 385-392.

[231] J. H. Chen, W. Luo, Z. X. Chen, S. C. Yan, F. Xu, and Y.-Q. Lu, "Mechanical modulation of a hybrid graphene-microfiber structure," Advanced Optical Materials, 2016, 4(6): 853-857.

[232] G. X. Ni, H. Z. Yang, W. Ji, S. J. Baeck, C. T. Toh, J. H. Ahn, et al., "Tuning optical conductivity of large-scale CVD graphene by strain engineering," Advanced Materials, 2014, 26(7): 1081-1086.

[233] V. M. Pereira, R. M. Ribeiro, N. M. R. Peres, and A. H. Castro Neto, "Optical properties of strained graphene," Europhysics Letters, 2011, 92(6): 67001.

[234] Q. Sun, X. Sun, W. Jia, Z. Xu, H. Luo, D. Liu, et al., "Graphene-assisted microfiber for optical-powerbased temperature sensor," IEEE Photonics Technology Letters, 2015, 28(4): 383-386.

[235] X. C. Yu, Y. Zhi, S. J. Tang, B. B. Li, Q. Gong, C. W. Qiu, et al., "Optically sizing single atmospheric particulates with a 10 -nm resolution using a strong evanescent field," Light: Science \& Applications, 2018, 7(4): 18003.

[236] F. Gao, H. Liu, C. Sheng, C. Zhu, and S. N. Zhu, "Refractive index sensor based on the leaky radiation of a microfiber," Optics Express, 2014, 22(10): 12645-12652.

[237] Z. Zhang, J. Pan, Y. Tang, Y. Xu, L. Zhang, Y. Gong, et al., "Optical micro/nanofibre embedded soft film enables multifunctional flow sensing in microfluidic chips," Lab on a Chip, 2020, 20: 2572-2579.

[238] P. Polynkin, A. Polynkin, N. Peyghambarian, and M. Mansuripur, "Evanescent field-based optical fiber sensing device for measuring the refractive index of liquids in microfluidic channels," Optics Letters, 2005, 30(11): 1273-1275.

[239] G. Liu, Y. Wu, K. Li, P. Hao, P. Zhang, and M. Xuan, "Mie scattering-enhanced fiber-optic refractometer," IEEE Photonics Technology Letters, 2012, 24(8): 658-660.

[240] Y. Zhi, X. C. Yu, Q. Gong, L. Yang, and Y. F. Xiao, "Single nanoparticle detection using optical microcavities," Advanced Materials, 2017, 29(12): 1604920.

[241] X. Jolly, V. Serge, M. Fabian, and V. Frank, "Advances in optoplasmonic sensors - combining optical nano/microcavities and photonic crystals with plasmonic nanostructures and nanoparticles," Nanophotonics, 2018, 7(1): 1-38.

[242] S. Wang, X. Pan, and L. Tong, "Modeling of nanoparticle-induced Rayleigh-Gans scattering for nanofiber optical sensing," Optics Communications, 2007, 276(2): 293-297.

[243] S. J. Tang, S. Liu, X. C. Yu, Q. Song, Q. Gong, and Y. F. Xiao, "On-chip spiral waveguides for ultrasensitive and rapid detection of nanoscale objects," Advanced Materials, 2018, 30(25): 1800262.

[244] Y. L. Chen and Q. Gong, "Hybrid plasmonic-photonic mode in a subwavelength fiber for enhanced single-nanoparticle detection," Physical Review A, 2015, 91(1): 013805.

[245] X. C. Yu, B. B. Li, P. Wang, L. Tong, X. F. Jiang, Y. $\mathrm{Li}$, et al., "Single nanoparticle detection and sizing using a nanofiber pair in an aqueous environment," Advanced Materials, 2014, 26(44): 7462-7467.

[246] Z. Wei, Z. Song, X. Zhang, and Z. Meng, "Microparticle detection with optical microfibers," IEEE Photonics Technology Letters, 2013, 25(6): 568-571.

[247] J. C. Beugnot, S. Lebrun, G. Pauliat, H. Maillotte, V. Laude, and T. Sylvestre, "Brillouin light scattering from surface acoustic waves in a subwavelength-diameter optical fibre," Nature Communications, 2014, 5(1): 5242.

[248] F. Xu, Z. X. Wu, and Y. Q. Lu, "Nonlinear optics in optical-fiber nanowires and their applications," Progress in Quantum Electronics, 2017, 55: 35-51.

[249] S. D. Emami, L. H. Jing, M. M. Rahman, F. Abdullah, H. A. Abdul-Rashid, M. M. Dashtabi, et $a l$,, "Evolution of surface acoustic waves in an optical microfiber," IEEE Journal of Quantum Electronics, 2017, 53(5): 1-8.

[250] O. Florez, P. F. Jarschel, Y. a. V. Espinel, C. M. B. Cordeiro, T. P. Mayer Alegre, G. S. Wiederhecker, et al., "Brillouin scattering self-cancellation," Nature Communications, 2016, 7(1): 1-8.

[251] Y. C. Shi, W. Luo, F. Xu, and Y. Q. Lu, "Photon-phonon interaction in a microfiber induced by optical and electrostrictive forces," Scientific Reports, 2017, 7: 41849.

[252] M. Cao, H. Li, M. Tang, Y. Mi, L. Huang, and G. Ren, "Forward stimulated Brillouin scattering in optical nanofibers," Journal of the Optical Society of America B, 2019, 36(8): 2079-2086.

[253] L. Huang, Y. Zhang, Y. Cui, J. Qiu, and X. Liu, "Microfiber-assisted gigahertz harmonic mode-locking in ultrafast fiber laser," Optics Letters, 2020, 45(17): 4678-4681.

[254] A. Godet, A. Ndao, T. Sylvestre, V. Pecheur, S. Lebrun, G. Pauliat, et al., "Brillouin spectroscopy of optical microfibers and nanofibers," Optica, 2017, 4(10): 1232-1238.

[255] J. Huang, X. Zhong, H. Liang, L. Cheng, J. Li, and B. Guan, "Brillouin scattering from hybrid acoustic wave in a microscaled fiber for gas pressure sensing," IEEE Photonics Journal, 2017, 9(2): 1-6. 
[256] W. Luo, H. Q. Cao, Y. K. Dong, Y. Q. Lu, F. Xu, and G. Brambilla, "Evolution and spatial distribution of Brillouin backscattering associated to hybrid acoustic modes in sub-wavelength silica microfibers," arXiv preprint: 1807.02774 (2018).

[257] C. Huang, H. Sun, H. Liang, L. Cheng, L. Chen, X. Bao, et al., "Refractive index sensing based on Brillouin scattering in a micro fiber," Applied Physics Express, 2019, 12(8): 082013.

[258] J. P. Dakin, D. J. Pratt, G. W. Bibby, and J. N. Ross, "Distributed optical fibre Raman temperature sensor using a semiconductor light source and detector," Electronics Letters, 1985, 21(13): 569-570.

[259] A. Ukil, H. Braendle, and P. Krippner, "Distributed temperature sensing: review of technology and applications," IEEE Sensors Journal, 2012, 12(5): 885-892.

[260] L. Shan, G. Pauliat, G. Vienne, L. Tong, and S. Lebrun, "Design of nanofibres for efficient stimulated Raman scattering in the evanescent field," Journal of the European Optical Society Rapid Publications, 2013, 8: 13030

[261] L. Shan, G. Pauliat, G. Vienne, L. Tong, and S. Lebrun, "Stimulated Raman scattering in the evanescent field of liquid immersed tapered nanofibers," Applied Physics Letters, 2013, 102(20): 201110.

[262] C. Wang, L. Zeng, Z. Li, and D. Li, "Review of optical fibre probes for enhanced Raman sensing," Journal of Raman Spectroscopy, 2017, 48(8): 1040-1055.

[263] J. Cao, D. Zhao, X. Lei, Y. Liu, and Q. Mao, "One-pot hydrothermal synthesis of silver nanoplates on optical fiber tip for surface-enhanced Raman scattering," Applied Physics Letters, 2014, 104(20): 201906.

[264] J. Zhang, S. Chen, T. Gong, X. Zhang, and Y. Zhu, "Tapered fiber probe modified by Ag nanoparticles for SERS detection," Plasmonics, 2016, 11(3): 743-751.

[265] Z. Huang, X. Lei, Y. Liu, Z. Wang, X. Wang, Z. Wang, et al., "Tapered optical fiber probe assembled with plasmonic nanostructures for surface-enhanced Raman scattering application," ACS Applied Materials \& Interfaces, 2015, 7(31): 17247-17254.

[266] W. Zhang, Z. Fang, and X. Zhu, "Near-field Raman spectroscopy with aperture tips," Chemical Reviews, 2017, 117(7): 5095-5109.

[267] B. L. Li, D. R. Li, J. H. Chen, Z. Y. Liu, G. H. Wang, X. P. Zhang, et al., "Hollow core micro-fiber for optical wave guiding and microfluidic manipulation," Sensors and Actuators B: Chemical, 2018, 262: 953-957.

[268] A. Stiebeiner, O. Rehband, R. Garcia-Fernandez, and A. Rauschenbeutel, "Ultra-sensitive fluorescence spectroscopy of isolated surface-adsorbed molecules using an optical nanofiber," Optics Express, 2009, 17(24): 21704-21711.

[269] Z. Li, Y. Xu, W. Fang, L. Tong, and L. Zhang, "Ultra-sensitive nanofiber fluorescence detection in a microfluidic chip," Sensors, 2015, 15(3): 4890-4898.

[270] V. S. Afshar, S. C. Warren-Smith, and T. M. Monro, "Enhancement of fluorescence-based sensing using microstructured optical fibres," Optics Express, 2007, 15(26): 17891-17901.

[271] S. C. Warren-Smith, S. Afshar, and T. M. Monro, "Fluorescence-based sensing with optical nanowires: a generalized model and experimental validation," Optics Express, 2010, 18(9): 9474-9485.

[272] F. Le Kien, S. D. Gupta, V. I. Balykin, and K. Hakuta, "Spontaneous emission of a cesium atom near a nanofiber: Efficient coupling of light to guided modes," Physical Review A, 2005, 72(3): 032509.

[273] H. Nha and W. Jhe, "Cavity quantum electrodynamics for a cylinder: Inside a hollow dielectric and near a solid dielectric cylinder," Physical Review A, 1997, 56(3): 2213-2220.

[274] V. V. Klimov and M. Ducloy, "Spontaneous emission rate of an excited atom placed near a nanofiber," Physical Review A, 2004, 69(1): 013812.

[275] T. Vo-Dinh and P. Kasili, "Fiber-optic nanosensors for single-cell monitoring," Analytical and Bioanalytical Chemistry, 2005, 382(4): 918-925.

[276] Q. Yang, H. Wang, S. Chen, X. Lan, H. Xiao, H. Shi, et al., " Fiber-optic-based micro-probe using hexagonal 1-in-6 fiber configuration for intracellular single-cell pH measurement," Analytical Chemistry, 2015, 87(14): 7171-7179.

[277] W. Tan, Z. Y. Shi, S. Smith, D. Birnbaum, and R. Kopelman, "Submicrometer intracellular chemical optical fiber sensors," Science, 1992, 258(5083): 778-781.

[278] J. Lee, H. R. Lee, J. Pyo, Y. Jung, J. Y. Seo, H. G. Ryu, et al., "Quantitative probing of $\mathrm{Cu}^{2+}$ ions naturally present in single living cells," Advanced Materials, 2016, 28(21): 4071-4076.

[279] T. Vo-Dinh and Y. Zhang, "Single-cell monitoring using fiberoptic nanosensors," WIREs Nanomedicine and Nanobiotechnology, 2011, 3(1): 79-85.

[280] Y. Xu, W. Fang, and L. Tong, "Real-time control of micro/nanofiber waist diameter with ultrahigh accuracy and precision," Optics Express, 2017, 25(9): 10434-10440.

[281] L. Xiao, M. D. Grogan, S. G. Leon-Saval, R. Williams, R. England, W. J. Wadsworth, et al., 
"Tapered fibers embedded in silica aerogel," Optics Letters, 2009, 34(18): 2724-2726.

[282] L. Xiao, M. D. W. Grogan, W. J. Wadsworth, R. England, and T. A. Birks, "Stable low-loss optical nanofibres embedded in hydrophobic aerogel," Optics Express, 2011, 19(2): 764-769.

[283] F. Xu, V. Pruneri, V. Finazzi, and G. Brambilla, “An embedded optical nanowire loop resonator refractometric sensor," Optics Express, 2008, 16(2): 1062-1067.

[284] W. Jin, H. Xuan, C. Wang, W. Jin, and Y. Wang, "Robust microfiber photonic microcells for sensor and device applications," Optics Express, 2014, 22(23): 28132-28141.

[285] J. Zhu, X. Liu, Q. Shi, T. He, Z. Sun, X. Guo, et al., "Development trends and perspectives of future sensors and MEMS/NEMS," Micromachines, 2020, 11(1): 7.

[286] R. Soref, "Silicon photonics: A review of recent literature," Silicon, 2010, 2(1): 1-6.

[287] E. Luan, H. Shoman, D. M. Ratner, K. C. Cheung, and L. Chrostowski, "Silicon photonic biosensors using label-free detection," Sensors, 2018, 18(10): 3519.

[288] J. H. Li, J. H. Chen, and F. Xu, "Sensitive and wearable optical microfiber sensor for human health monitoring," Advanced Materials Technologies, 2018, 3(12): 1800296.

[289] L. Zhang, J. Pan, Z. Zhang, H. Wu, N. Yao, D. Cai, et al., "Ultrasensitive skin-like wearable optical sensors based on glass micro/nanofibers," Opto-Electronic Advances, 2020, 3(03): 190022. 